\title{
A New Calibration Method for a Directly Driven 3PRR Positioning System
}

\author{
Jia-Si Mo • Zhi-Cheng Qiu • Lei Zeng • \\ Xian-Min Zhang
}

Received: 28 February 2016 / Accepted: 4 July 2016 / Published online: 5 August 2016

(C) The Author(s) 2016. This article is published with open access at Springerlink.com

\begin{abstract}
Calibration is one of the most important works for the parallel manipulator. The manufacturing and assembling errors will modify the designed parameters of the parallel mechanism, leading to the positioning errors. Calibration is an effective method for improving the accuracy of the parallel mechanism. It is vital to identify the parameters and calibrate the system aiming at improving the positioning accuracy. In order to build an object stage of the micro/nano operation system, a 3 degree-of-freedoms (DOFS) parallel mechanism has been designed and constructed, with combination of legs of the PRR type (the underline of the $\mathrm{P}$ represent the actuated joint), $\mathrm{P}$ and $\mathrm{R}$ representing prismatic and revolute pairs respectively (3PRR). Due to the space constraint, this 3 PRR mechanism is built without the end-effector feedback, and must be calibrated for high accuracy positioning. The error model of the 3 PRR mechanism has been derived and analyzed, and the error distribution mappings of the 3PRR mechanism are obtained. The calibration method based on the error model is investigated. Since some parameters are difficult to
\end{abstract}

\footnotetext{
J.-S. Mo · Z.-C. Qiu · L. Zeng · X.-M. Zhang ( $\bowtie)$ Guangdong Province Key Laboratory of Precision and Manufacturing Technology, South China University of Technology, Guangzhou 510641, China

e-mail: zhangxm@scut.edu.cn

J.-S. Mo

e-mail: mo.jiasi@mail.scut.edu.cn

Z.-C. Qiu

e-mail: zhchqiu@scut.edu.cn
}

be identified by using the decoupling error model, the assistant measurements are proposed and utilized to compensate for this calibration method. Numerical simulations and experiments are carried out. The simulation results show that it is not enough to calibrate this system by using the calibration method based on error model only, and the experimental results demonstrate that the combined assistant measurements will achieve a better effect for calibration.

Keywords Calibration method - Ultrasonic linear motor $\cdot 3$ PRR positioning system $\cdot$ Parallel mechanism

\section{Introduction}

Parallel mechanisms are becoming more and more popular in application fields due to the high precision, high speed, and high load capacity [1]. Micro/nano operating system is an important part in the field of precision operation and manufacturing. The object stage of the micro/nano operating system is used to place and move samples at large-scale stroke. Therefore the object stage needs to possess the features of large stroke and high precision positioning. Macromicro combination method can make up the stroke scope of the micro positioning mechanism, whereas parallel mechanisms can realize high precision positioning. Hence, under the environment of the scanning electron microscopy (SEM), the 3 degree-of-freedoms (DOFS) parallel mechanism with combination of legs of the PRR type (the underline of the $\underline{P}$ represent the actuated joint), $P$ and $R$ representing prismatic and 
revolute pairs respectively ( $3 \underline{P R R}$ ) has been designed and constructed. The 3 PRR parallel positioning mechanism is adopted to constitute the macro-movement part of the macro-micro combination precision positioning system [2].

Although the parallel mechanisms are more accurate than the serial mechanisms theoretically [3], they have some similar problems which affect the positioning accuracy. The major part of the mechanism is usually a semi closed-loop mechanism with the actuated joint feedback only, due to the cost restriction. Although the actuated joints have the capacity to accomplish positioning very well [4], the transmission chains usually introduce some nonlinear characteristics, such as backlash, friction, elastic deformation, etc. These nonlinear characteristics will lead to the positioning errors of the end-effector [5]. As the actuated joint feedback control method only has the state information of the actuated joints, it is hardly to control the end-effector well without the state information of the end-effector. If an observer is designed to estimate the end-effector state information [6] the observation noise and errors will be introduced, which is not suitable for high accurate positioning. It is an ideal way to solve the high accurate positioning problem by building a full closed-loop system with end-effect sensors, but the DOFS of the sensor measurement is limited. The suitable sensor is seldom to detect the multiple DOFS mechanism [7]. For a mechanism without a suitable sensor for the end-effector feedback but requested to fulfill the high accurate positioning, the calibration becomes an important and effective method to ensure the positioning accuracy. References $[8,9]$ adopted the camera as the sensor to measure the end-effector state information, but the field of view and the pixel size are limited in the machine vision method It is difficult to reach micron level precision in real time by using the camera as sensor.

Calibration and control are two methods for improving the accuracy of the mechanism in different aspects. For a mechanism without the end-effector feedback, calibration will be the preferred way to improve the accuracy. The parameters identification and system calibration are vital for the high accuracy positioning system. Without calibration, the control will be helpless. The calibration method always based on the error modeling of the mechanism, and the accuracy of the model affect the accuracy of the calibration directly. Shao used the closed-loop kinematic chain model to obtain the error model of the $3 \underline{R R R}$ mechanism [10]. Yang used the coordinate conversion to derive the error model of the Delta mechanism [11]. These error models are always based on the geometry relationship and the differential kinematics [12]. Derivation of the error model based on the first order Taylor Expansion the higher order terms are usually eliminated. But the higher order terms are always the coupling terms of the errors eliminating those means decoupling the affections of the errors. For the serial mechanism, it is not a big problem, because the serial mechanisms are decoupling essentially. But for the parallel mechanism, the coupling of the input and output exists in the system. Decoupling means that some of the coupling errors will no longer be considered, and the modeling errors will be caused.

There are few people consider the affections of the error decoupling on parallel mechanism, because the coupling error model is too complex. The coupling error model is hardly to be transferred to the identified model, so it is not convenient for identifying the system parameters. There are some problems in parameters identification. Nubiola identified the parameters by solving the overdetermined equation set [13], but the overdetermined equation is unable to get an analytical solution. The approximate solution can only be obtained. Different optimization algorithm will get different results in solving the overdetermined equation set. Jiménez identified the parameters with the least square method and calibrated the system with the least square solution [14], but the least square is not the optimal estimation. Xin identified the 3 DOFS mechanism with the iterative algorithm based on the forward kinematic [15] and it is hardly extended to the mechanism without the closedformed forward kinematic solutions. Nubiola compared with different sensor calibration results, but the calibration model is the same [16]. Wang used the unit quaternion form to calibrate the 6 DOFs robot [17], but it is too complex for the planar parallel mechanisms. For calibration purpose, the most concerned is to find out the main error source which will significantly affect the positioning accuracy and must be calibrated. The calibration method based on solving the overdetermined equation set is unable to identify all the parameters accurately, due to the math nature of the overdetermined equation set. If the main error source is identified difficulty, the assistant measurements are needed to compensate for the calibration. 


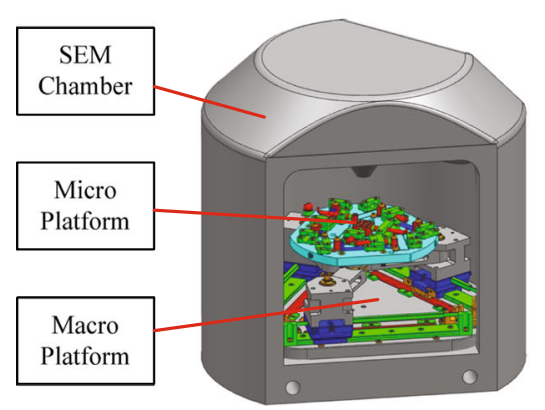

(a) Macro-micro combination object stage in SEM chamber;

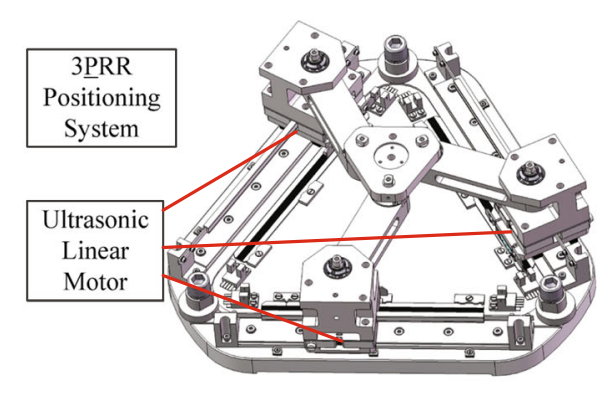

(b) Macro movement part (3ㄹRR positioning system);

Fig. $13 \mathrm{D}$ diagram of $3 \underline{P R R}$ positioning system

In order to build an object stage of the SEM, the 3PRR planar parallel mechanism has been designed and built It is designed to fulfill micron grade positioning with the millimeter travel stroke. After the research of calibrating the system, it is found that the decoupling error model will cause some parameters unable to be identified, and some of these unidentified parameters are the main error sources. Considering that the coupling error model is too complex to use and the decoupling error model is not ideal for high accurate calibration, a new calibration method has been proposed.

The rest of this article is organized as follows. The error model is derived and the main error sources are obtained in Section 2. In Section 3 the calibration methods based on the decoupling error model are analyzed, and the assistant measurement is used to compensate for the calibration based on error model. Section 4 conducts the experiments, and the experimental results verify this new calibration method. Conclusions are drawn in Section 5.

\section{Error Modeling and Analysis}

In order to build up a macro-micro combination object stage of the SEM, a 3PRR planar parallel mechanism is designed and constructed. The $3 \mathrm{D}$ diagram is shown in Fig. 1. Magnetic field free and compact structure is two essential requirements under the SEM environment. This 3PRR planar parallel mechanism driven by ultrasonic linear motors is able to meet these two requirements. Because direct drive method is an effective method to minimize the nonlinear characteristics of the mechanism this $3 \underline{P R R}$ is constructed using the direct drive mechanism for the high accuracy positioning. However, in practical work
Fig. 2 Reference frame of the 3 PRR

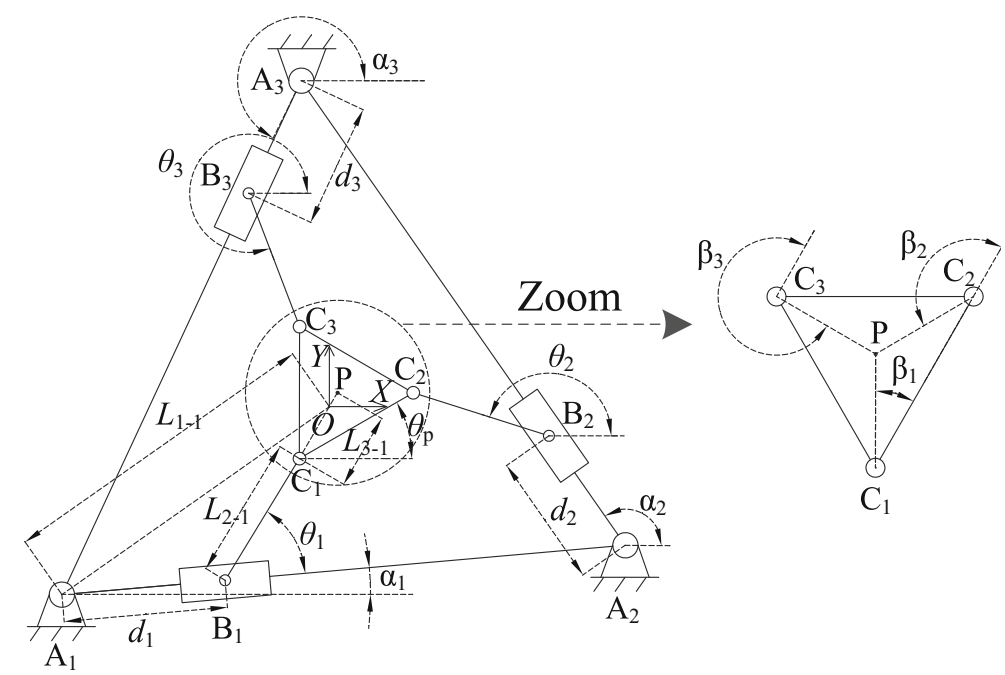


there are many errors introduced without the endeffector feedback, which limited the performance of the positioning accuracy.

The errors in this system include the certain errors and uncertain errors. The certain errors like the manufacturing and assembling errors are able to be identified; but the uncertain errors like the disturbances and noise are difficult to be identified. The certain error model of the mechanism is based on the differential kinematics relationship and the uncertain error model is based on the statistics and probability theory.

\subsection{Error Modeling}

Here, the certain error of the kinematic model is mainly considered. At first, building reference coordinate of the 3PRR in Fig. 2.

The 3PRR positioning system uses the linkage to connect the static platform $\left(\triangle \mathrm{A}_{1} \mathrm{~A}_{2} \mathrm{~A}_{3}\right)$ and moving platform $\left(\Delta \mathrm{C}_{1} \mathrm{C}_{2} \mathrm{C}_{3}\right)$ symmetrically. The static platform and the moving platform are all equilateral triangle with the circumradius of $\mathrm{L}_{1}=210 \mathrm{~mm}, \mathrm{~L}_{3}=$ $28 \mathrm{~mm}$. The sliders $\mathrm{B}_{1}, \mathrm{~B}_{2}, \mathrm{~B}_{3}$ on the sides $\left(\mathrm{A}_{1} \mathrm{~A}_{2}\right.$, $\mathrm{A}_{2} \mathrm{~A}_{3}, \mathrm{~A}_{3} \mathrm{~A}_{1}$ ) of the static platform forms the prismatic actuated joints The actuated distances are $d_{1}$, $d_{2}$ and $d_{3}$. The sliders $\mathrm{B}_{i}(i=1,2,3)$ connect to the nodes $\mathrm{C}_{i}(i=1,2,3)$ of the moving platform with the linkages in equal length $\left(\mathrm{B}_{1} \mathrm{C}_{1}=\mathrm{B}_{2} \mathrm{C}_{2}=\right.$
$\mathrm{B}_{3} \mathrm{C}_{3}=\mathrm{L}_{2}=95 \mathrm{~mm}$ ). Both ends of the linkages are revolute pairs, composing of the passive joints of the 3PRR. The node P: $\left(x_{\mathrm{p}} y_{\mathrm{p}}\right)$ is the geometric center of the moving platform and defined as the end-effector point of this system. The $\mathrm{O}-\mathrm{XY}$ is the global coordinate, and $\theta_{1} \theta_{2}, \theta_{3}$ are included angles between the sides $\mathrm{B}_{1} \mathrm{C}_{1}, \mathrm{~B}_{2} \mathrm{C}_{2}, \mathrm{~B}_{3} \mathrm{C}_{3}$ and $\mathrm{X}$-axis, respectively. $\theta_{\mathrm{p}}$ is the included angle between the side $\mathrm{C}_{1} \mathrm{C}_{2}$ and $\mathrm{X}$-axis.

Using the closed-loop vector relationship, the equation is given

$$
\mathbf{A}_{i} \mathbf{B}_{i}+\mathbf{B}_{i} \mathbf{C}_{i}+\mathbf{C}_{i} \mathbf{P}+\mathbf{P O}+\mathbf{O A}_{i}=\mathbf{0},(i=1,2,3) .
$$

Expanding (1) into projections of two axes (X-axis, Y-axis) the kinematic model of the $3 \mathrm{PRR}$ is

$$
\left\{\begin{array}{c}
x_{\mathrm{p}}=d_{i} \cos \alpha_{i}+\mathrm{L}_{2-i} \cos \theta_{i}+\mathrm{L}_{3-i} \cos \left(\gamma_{i}\right)+x_{\mathrm{A} i} \\
y_{\mathrm{p}}=d_{i} \sin \alpha_{i}+\mathrm{L}_{2-i} \sin \theta_{i}+\mathrm{L}_{3-i} \sin \left(\gamma_{i}\right)+y_{\mathrm{A} i}
\end{array}\right.
$$

where $\alpha_{i}=2 \pi(i-1) / 3 ; \gamma_{i}=\beta_{i}+\theta_{\mathrm{p}} ; \beta_{i}=\pi / 6+\alpha_{i}$; $x_{\mathrm{A} i}$ and $y_{\mathrm{A} i}$ are related to the $\alpha_{i}$ and $\mathrm{L}_{1-i}$, respectively $(i=1,2,3)$.

One can introduce a small perturbation $(\delta)$ into all active error sources. Because the error $\delta \theta_{i}$ is caused by the other error sources, it is a passive error instead of an active error The error $\delta \theta_{i}$ becomes a redundancy term and it is not considered in the error modeling. The kinematic model with errors can be obtained as follow.

$\left\{\begin{array}{c}x_{\mathrm{p}}+\delta x_{\mathrm{p}}=\left(d_{i}+\delta d_{i}\right) \cos \left(\alpha_{i}+\delta \alpha_{i}\right)+\left(\mathrm{L}_{2-i}+\delta \mathrm{L}_{2-i}\right) \cos \left(\theta_{i}\right)+\left(\mathrm{L}_{3-i}+\delta \mathrm{L}_{3-i}\right) \cos \left(\gamma_{i}+\delta \gamma_{i}\right)+\left(x_{\mathrm{A} i}+\delta x_{\mathrm{A} i}\right) \\ y_{\mathrm{p}}+\delta y_{\mathrm{p}}=\left(d_{i}+\delta d_{i}\right) \sin \left(\alpha_{i}+\delta \alpha_{i}\right)+\left(\mathrm{L}_{2-i}+\delta \mathrm{L}_{2-i}\right) \sin \left(\theta_{i}\right)+\left(\mathrm{L}_{3-i}+\delta \mathrm{L}_{3-i}\right) \sin \left(\gamma_{i}+\delta \gamma_{i}\right)+\left(y_{\mathrm{A} i}+\delta y_{\mathrm{A} i}\right)\end{array}\right.$,

where $\gamma_{i}+\delta \gamma_{i}=\beta_{i}+\delta \beta_{i}+\theta_{\mathrm{p}}+\delta \theta_{\mathrm{p}}(i=1,2,3)$; and the error sources are listed in Table 1 .

Using the approximate formula $\left\{\begin{array}{l}\sin x \approx x \\ \cos x \approx 1\end{array}\right.$, higher order terms and substituting Eq. 3 into Eq. 2, the simplified error model of the 3PRR is obtained as

$(x \ll 1)$ to simplify the error model, rounding off the

$\left\{\begin{array}{l}\delta x_{\mathrm{p}}=-\left(d_{i} \sin \alpha_{i}\right) \delta \alpha_{i}+\left(\cos \alpha_{i}\right) \delta d_{i}+\left(\cos \theta_{i}\right) \delta \mathrm{L}_{2-i}-\left(\mathrm{L}_{3-i} \sin \gamma_{i}\right) \delta \gamma_{i}+\left(\cos \gamma_{i}\right) \delta \mathrm{L}_{3-i}+\delta x_{\mathrm{A} i} \\ \delta y_{\mathrm{p}}=\left(d_{i} \cos \alpha_{i}\right) \delta \alpha_{i}+\left(\sin \alpha_{i}\right) \delta d_{i}+\left(\sin \theta_{i}\right) \delta \mathrm{L}_{2-i}+\left(\mathrm{L}_{3-i} \cos \gamma_{i}\right) \delta \gamma_{i}+\left(\sin \gamma_{i}\right) \delta \mathrm{L}_{3-i}+\delta y_{\mathrm{A} i}\end{array},(i=1,2,3)\right.$.

Equation 4 shows that the end-effector positioning errors $\left(\delta x_{\mathrm{p}} \delta y_{\mathrm{p}}\right)$ are coupling with the orientation error $\delta \theta_{\mathrm{p}}$. In order to decoupling the Eq. 4, one can multiply $\delta x_{\mathrm{p}}$ and $\delta y_{\mathrm{p}}$ with $\cos \theta_{i}$ and $\sin \theta_{i}$ respectively as

$\left\{\begin{array}{l}\cos \theta_{i} \delta x_{\mathrm{p}}=\cos \theta_{i}\left[-\left(d_{i} \sin \alpha_{i}\right) \delta \alpha_{i}+\left(\cos \alpha_{i}\right) \delta d_{i}+\left(\cos \theta_{i}\right) \delta \mathrm{L}_{2-i}-\left(\mathrm{L}_{3-i} \sin \gamma_{i}\right) \delta \gamma_{i}+\left(\cos \gamma_{i}\right) \delta \mathrm{L}_{3-i}+\delta x_{\mathrm{A} i}\right] \\ \sin \theta_{i} \delta y_{\mathrm{p}}=\sin \theta_{i}\left[\left(d_{i} \cos \alpha_{i}\right) \delta \alpha_{i}+\left(\sin \alpha_{i}\right) \delta d_{i}+\left(\sin \theta_{i}\right) \delta \mathrm{L}_{2-i}+\left(\mathrm{L}_{3-i} \cos \gamma_{i}\right) \delta \gamma_{i}+\left(\sin \gamma_{i}\right) \delta \mathrm{L}_{3-i}+\delta y_{\mathrm{A} i}\right]\end{array},(i=1,2,3)\right.$. 
Equation 5 can be expressed in terms of the input errors and output errors as

$$
\begin{aligned}
& \cos \theta_{i} \delta x_{\mathrm{p}}+\sin \theta_{i} \delta y_{\mathrm{p}}+\mathrm{L}_{3-i} \sin \left(\gamma_{i}-\theta_{i}\right) \delta \theta_{\mathrm{p}} \\
= & -d_{i} \sin \left(\alpha_{i}-\theta_{i}\right) \delta \alpha_{i}+\cos \left(\alpha_{i}-\theta_{i}\right) \delta d_{i} \\
& -\mathrm{L}_{3-i} \sin \left(\gamma_{i}-\theta_{i}\right) \delta \beta_{i}+\cos \left(\gamma_{i}-\theta_{i}\right) \delta \mathrm{L}_{3-i} \\
& +\cos \theta_{i} \delta x_{\mathrm{A} i}+\sin \theta_{i} \delta y_{\mathrm{A} i}+\delta \mathrm{L}_{2-i},(i=1,2,3) .
\end{aligned}
$$

Equation 6 can also be written as the matrix form

$\delta \mathbf{X}=\mathbf{J} \cdot \delta \mathbf{d}$, where $\delta \mathbf{X}=\left[\begin{array}{ll}\delta x_{\mathrm{p}} \delta y_{\mathrm{p}} \delta \theta_{\mathrm{p}}\end{array}\right]^{\mathrm{T}} ; \quad \delta \mathbf{d}_{i}=$ $\left[\delta \alpha_{i} \delta d_{i} \delta \beta_{i} \delta \mathrm{L}_{3-i} \delta x_{\mathrm{A} i} \delta y_{\mathrm{A} i} \delta \mathrm{L}_{2-i}\right]^{\mathrm{T}} ; \delta \mathbf{d}$

$\left[\begin{array}{lll}\delta \mathbf{d}_{1} & \delta \mathbf{d}_{2} & \delta \mathbf{d}_{3}\end{array}\right]^{\mathrm{T}}$;

$\mathbf{J}=\mathbf{J}_{\mathbf{1}}^{-1} \mathbf{J}_{\mathbf{2}} ; \quad \mathbf{J}_{1}=\left[\begin{array}{ccc}\cos \theta_{1} & \sin \theta_{1} & \mathrm{~L}_{3-1} \sin \left(\gamma_{1}-\theta_{1}\right) \\ \cos \theta_{2} & \sin \theta_{2} & \mathrm{~L}_{3-2} \sin \left(\gamma_{2}-\theta_{2}\right) \\ \cos \theta_{3} & \sin \theta_{3} & \mathrm{~L}_{3-3} \sin \left(\gamma_{3}-\theta_{3}\right)\end{array}\right] ;$ $\mathbf{J}_{2}=\left[\begin{array}{ccc}\mathbf{A}_{11} & \mathbf{0}_{1 \times 7} & \mathbf{0}_{1 \times 7} \\ \mathbf{0}_{1 \times 7} & \mathbf{A}_{22} & \mathbf{0}_{1 \times 7} \\ \mathbf{0}_{1 \times 7} & \mathbf{0}_{1 \times 7} & \mathbf{A}_{33}\end{array}\right]_{3 \times 21} ;$

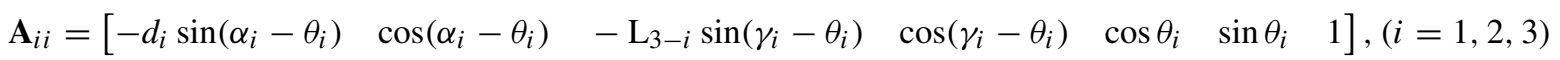

The matrix $\mathbf{J}$ is so call the error transfer matrix; the elements of the matrix $\mathbf{J}$ represent the contribution of every error sources to the end-effector errors.

\subsection{Analysis of the Error Sources}

Considering the error model of the $3 \underline{P R R}$, there are 21 error sources which will affect the positioning accuracy of the end-effector. Some of these error sources may affect the accuracy evidently, while the endeffector may be not so sensitive to the other error sources. The first row of the error transfer matrix $\mathbf{J}$ is corresponding to the end-effector error $\delta x_{\mathrm{p}}$; the second row corresponds to the end-effector error $\delta y_{\mathrm{p}}$, and the last row corresponds to $\delta \theta_{\mathrm{p}}$. For example, the elements of the first row in matrix $\mathbf{J}$ are the weights of the error sources. They determine the error sources in $\delta \mathbf{d}$ which will affect $\delta x_{\mathrm{p}}$ mostly. Put it another way, the percentage of the elements in $\mathbf{J}$ reflects the sensitivity of the error transfer. But in the whole workspace, every single point has a matrix $\mathbf{J}$, and the elements of the matrix $\mathbf{J}$ change in every positioning point. In order to figure out which source will affect the end-effector significantly in the whole workspace the sensitive factor of the errors in forms is defined as

$\mathrm{GESI}=\iint_{S}\left[\left(\mathbf{J}_{i j} \cdot \delta \mathbf{d}_{j}\right) / \delta \mathbf{X}_{i}\right] d s / \iint_{S} d s,(i=1,2,3 ; j=1$ to 21$)$,

where GESI (Global Errors Sensitive Index) is defined as the error transfer factor; $\mathbf{J}_{i j}$ is the element of the matrix $\mathbf{J} ; \delta \mathbf{d}_{j}$ is the error source corresponding to the $\mathbf{J}_{i j} ; \iint_{s} d s$ is the whole workspace area.

Equation 8 represents the global proportion of each error source. For a calculation example, the angle parameters of the 3PRR are described previously. The dimension parameters are $\mathrm{L}_{1}=210 \mathrm{~mm}, \mathrm{~L}_{2}=95 \mathrm{~mm}$, $\mathrm{L}_{3}=28 \mathrm{~mm}$. Assuming that all the angle errors are the same, and so does the length errors, the GESI of the 3PRR are shown in Figs. 3, 4 and 5.

The GESI histograms Figs. 3 and 4 specified the same Length errors and different angle errors; while Figs. 4 and 5 specified the same angle errors and different length errors.
Table 1 Description of Error source

\begin{tabular}{ll}
\hline Error sources, $(i=1,2,3)$ & Description \\
\hline$\delta d_{i}$ & Input errors of each actuated joint \\
$\delta \alpha_{i}$ & Angle errors of the static platform \\
$\delta \mathrm{L}_{2-i}$ & Errors of each linkage length \\
$\delta \mathrm{L}_{3-i}$ & Circumradius errors of the moving platform \\
$\delta \beta_{i}$ & Angle errors of the moving platform \\
$\delta x_{\mathrm{A} i}$ & Circumradius errors projection (Xaxis) of the static platform \\
$\delta y_{\mathrm{A} i}$ & Circumradius errors projection (Yaxis) of the static platform
\end{tabular}




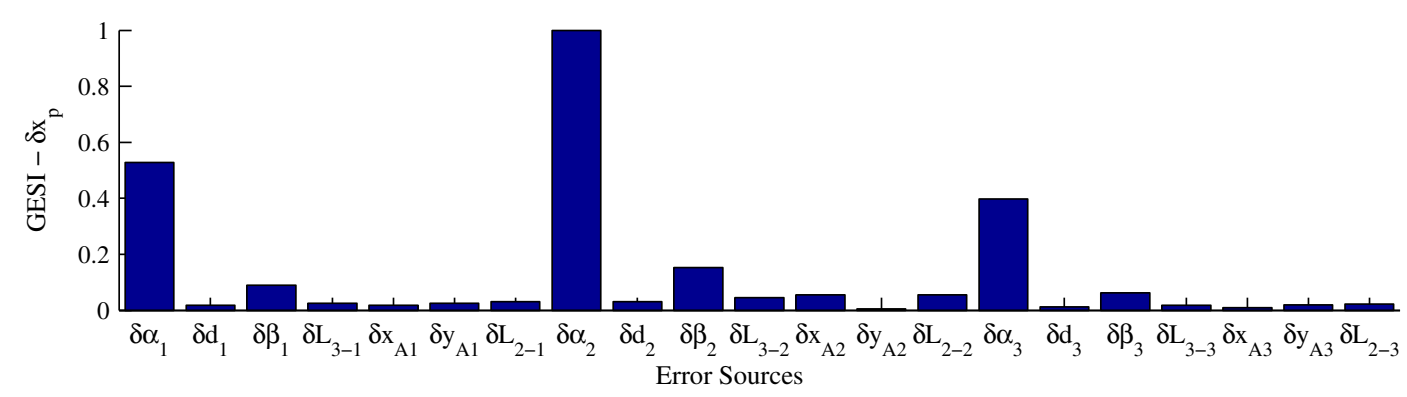

(a) The GESI to the $\delta x_{\mathrm{p}}$

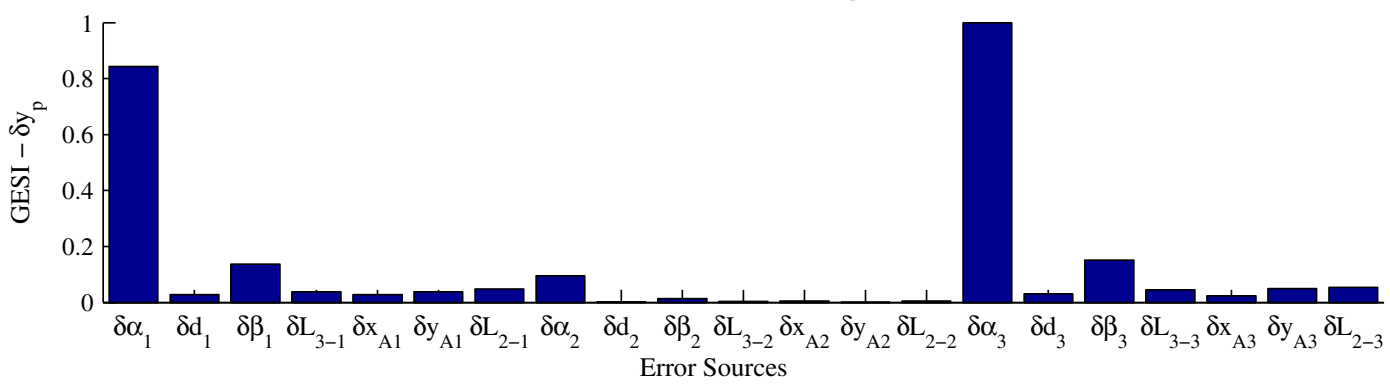

(b) The GESI to the $\delta y_{\mathrm{p}}$

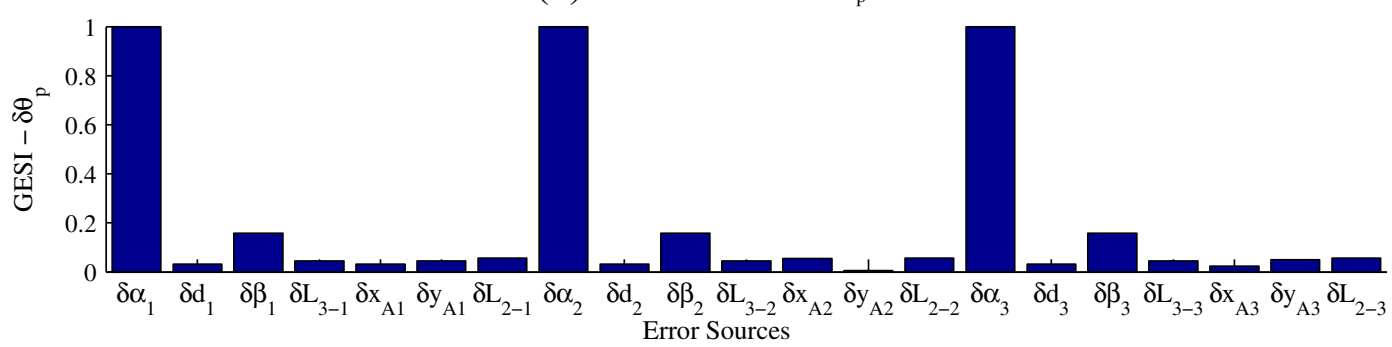

(c) The GESI to the $\delta \theta_{\mathrm{p}}$

Fig. 3 The 3PRR GESI of each error source (Angle errors are all specified as $0.1^{\circ}$; Length errors are all set as $0.01 \mathrm{~mm}$ )

After analyzing Figs. 3 and 4, the most sensitive error sources are the angles of the static platform $\left(\delta \alpha_{i}(i=1,2,3)\right)$ and the moving platform $\left(\delta \beta_{i}(i=\right.$ $1,2,3))$. These errors are certain errors coming from manufacturing and assembling. They are hardly to be eliminated, but they are able to be identified and calibrated. When the angle errors are at the same order of magnitude with length errors (Fig. 4), the other error sources also affect the end-effector obviously although $\delta \alpha_{i}(i=1,2,3)$ are the most sensitive error sources. However, if the magnitude of the angle errors is ten times higher than that of the length errors, the other effects are weakened, and $\delta \alpha_{i}(i=1,2,3)$ will become the main error sources.
After analyzing Figs. 4 and 5, the main error sources are different. This comparison specified the same angle errors, so the different of the main error sources caused by the different volume of the length errors. When the angle errors are at the same order of magnitude with length errors (Fig. 4), the main error sources are $\delta \alpha_{i}(i=1,2,3)$. But if the length errors are ten times higher than the angle errors, the main error sources will be changed to the linkage length errors. The comparisons of the Figs. 3, 4 and 5 are summarized in Table 2.

When the angle errors are multiplied with the radius, it will be amplified to arc errors Therefore, a little angle errors will cause remarkable end-effector 


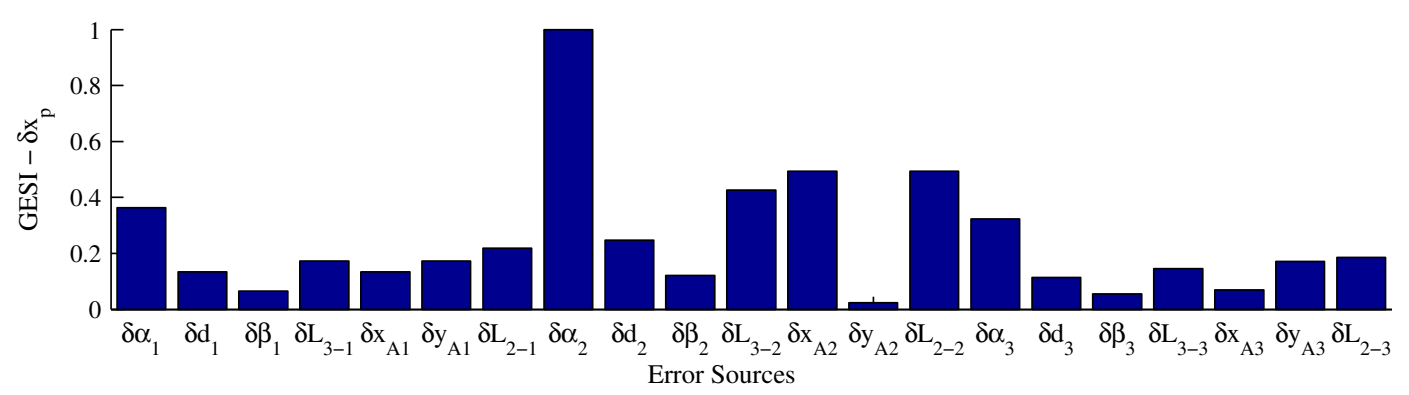

(a) The GESI to the $\delta x_{\mathrm{p}}$

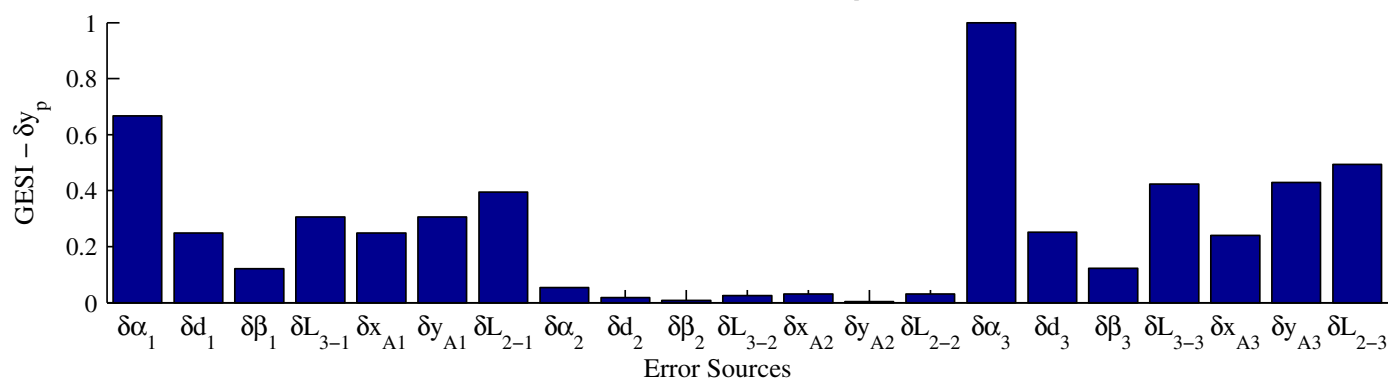

(b) The GESI to the $\delta y_{\mathrm{p}}$

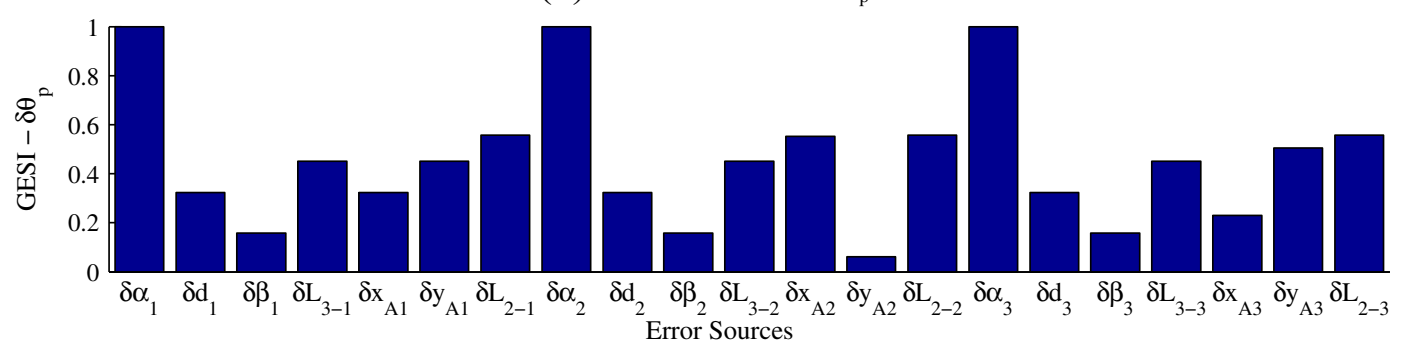

(c) The GESI to the $\delta \theta_{\mathrm{p}}$

Fig. 4 The 3PRR GESI of each error source (Angle errors are all specified as $0.01^{\circ}$; Length errors are all set as $0.01 \mathrm{~mm}$ )

errors. The length errors of the 3PRR result from the manufacturing, so they are usually less than $0.05 \mathrm{~mm}$. The angle errors $\delta \beta_{i}(i=1,2,3)$ also result from manufacturing, but it is not so sensitive to the end-effector errors. However the angle errors $\delta \alpha_{i}(i=1,2,3)$ are from the assembling, and it is much larger than $\delta \beta_{i}(i=1,2,3)$ and the installation angles are difficult to be guaranteed. The main error sources are related to the order of magnitude of the active errors. Because the angle errors (from assembling) are bigger than the length errors (from manufacturing) in practical situation, therefore, $\delta \alpha_{i}(i=1,2,3)$ are the main error sources of this 3PRR positioning system, and it is must be identified and calibrated.
2.3 The Relationship Between the Error Model and the Velocity Jacobian Matrix

The velocity Jacobian matrix is an important definition in robot field. It defines the velocity linear input-output equations relating the end-effector velocity vectors to the actuated joint variables [1], and the general form is

$\mathbf{A} \cdot \dot{\mathbf{X}}=\mathbf{B} \cdot \dot{\mathbf{d}}$

where $\dot{\mathbf{X}}$ is the output velocity vector of the endeffector, and $\dot{\mathbf{d}}$ is the input velocity vector of the actuated joint. 


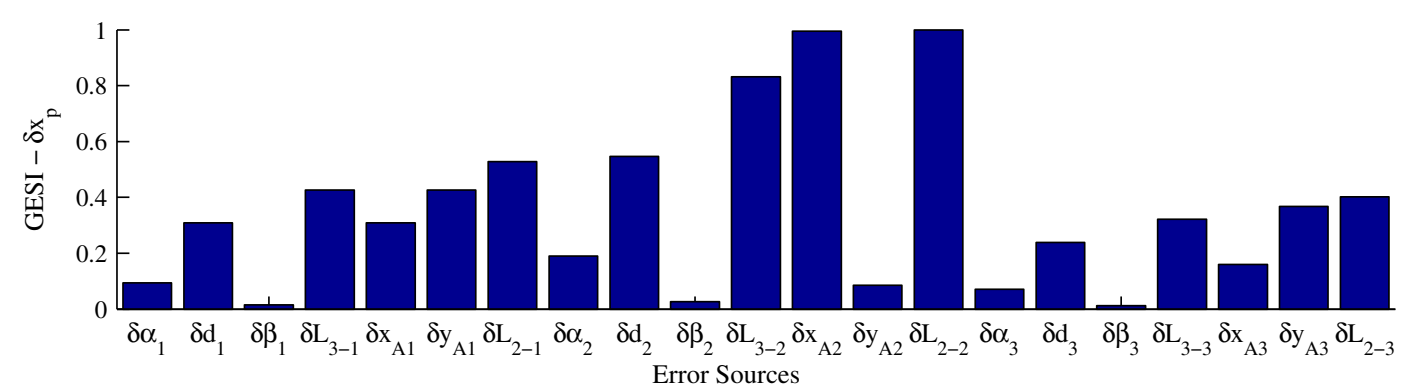

(a) The GESI to the $\delta x_{\mathrm{p}}$

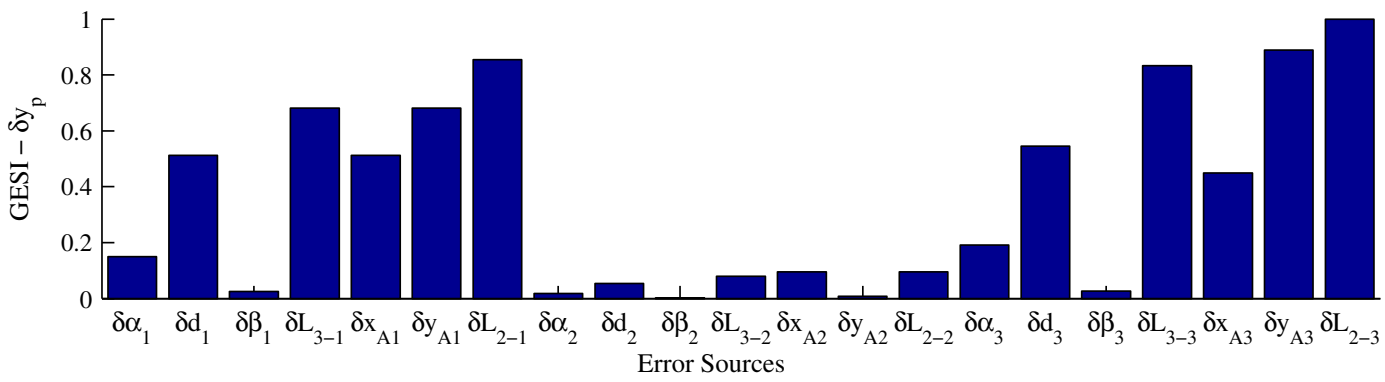

(b) The GESI to the $\delta y_{\mathrm{p}}$

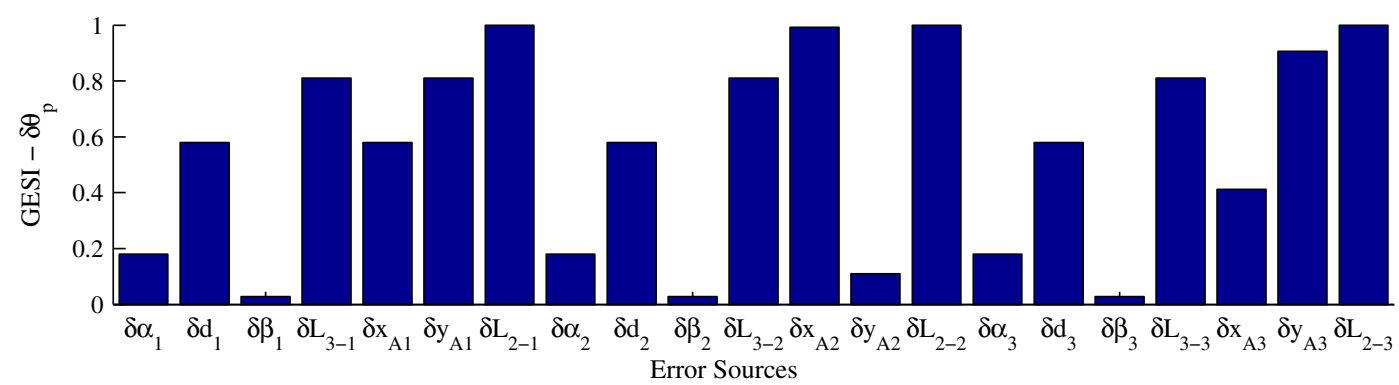

(c) The GESI to the $\delta \theta_{\mathrm{p}}$

Fig. 5 The 3PRR GESI of each error source (Angle errors are all specified as $0.01^{\circ}$; Length errors are all set as $0.1 \mathrm{~mm}$ )

The matrix $\mathbf{A}$ and $\mathbf{B}$ reflects the relationship between the input velocity and the output velocity. The matrix $\mathbf{A}$ and $\mathbf{B}$ can be combined and given as

$\mathbf{d} \doteq J_{\mathrm{inv}} \cdot \dot{\mathbf{X}}$,

where $\mathbf{J}_{\text {inv }}=\mathbf{B}^{-1} \cdot \mathbf{A}$

The matrix $\mathbf{J}_{\text {inv }}$ is called the inverse kinematic velocity Jacobian matrix. The $3 \underline{P} R R$ velocity Jacobian matrix has been derived by Jiasi Mo [2] and the equations of the 3 PRR velocity Jacobian matrix are

$$
\begin{aligned}
\mathbf{A} & =\left[\begin{array}{ccc}
\cos \theta_{1} & \sin \theta_{1} & \mathrm{~L}_{3} \sin \left(\pi / 6-\theta_{1}+\theta_{p}\right) \\
\cos \theta_{2} & \sin \theta_{2} & \mathrm{~L}_{3} \sin \left(\pi / 6-\theta_{2}+\theta_{p}\right) \\
\cos \theta_{3} & \sin \theta_{3} & \mathrm{~L}_{3} \sin \left(\pi / 6-\theta_{3}+\theta_{p}\right)
\end{array}\right], \\
\mathbf{B} & =\left[\begin{array}{ccc}
\cos \theta_{1} & 0 & 0 \\
0 & \cos \left(\theta_{2}-2 \pi / 3\right) & 0 \\
0 & 0 & \cos \left(\theta_{3}-4 \pi / 3\right)
\end{array}\right] .
\end{aligned}
$$

Table 2 The main error sources comparison

\begin{tabular}{lllll}
\hline Figure & Angle errors $/{ }^{\circ}$ & Length errors $/ \mathrm{mm}$ & Order of magnitude & Main error source \\
\hline Fig. 3 & 0.10 & 0.01 & Angle errors $>$ Length errors & Guide way angle error $\left(\delta \alpha_{i}\right)$ \\
Fig. 4 & 0.01 & 0.01 & Angle errors $=$ Length errors & Guide way angle error $\left(\delta \alpha_{i}\right)$ \\
Fig. 5 & 0.01 & 0.10 & Angle errors $<$ Length errors & Linkage length error $\left(\delta \mathrm{L}_{2-i} \delta \mathrm{L}_{3-i},, \delta x_{\mathrm{A} i} \delta y_{\mathrm{A} i}\right)$
\end{tabular}




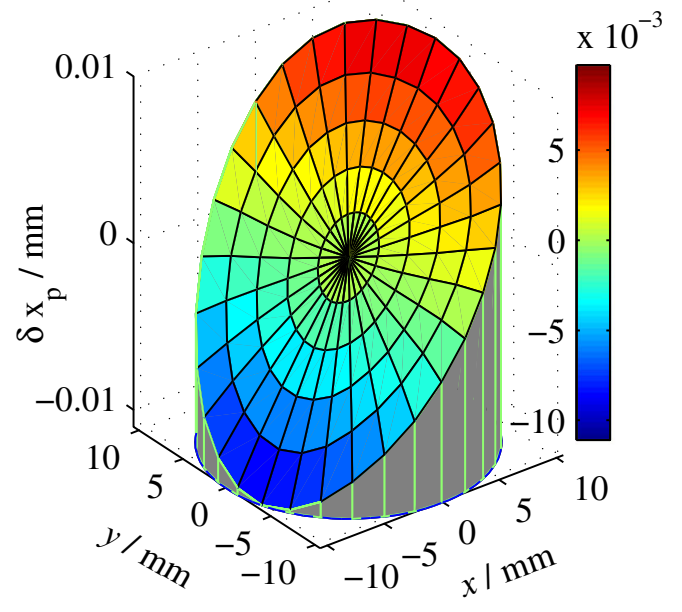

(a)

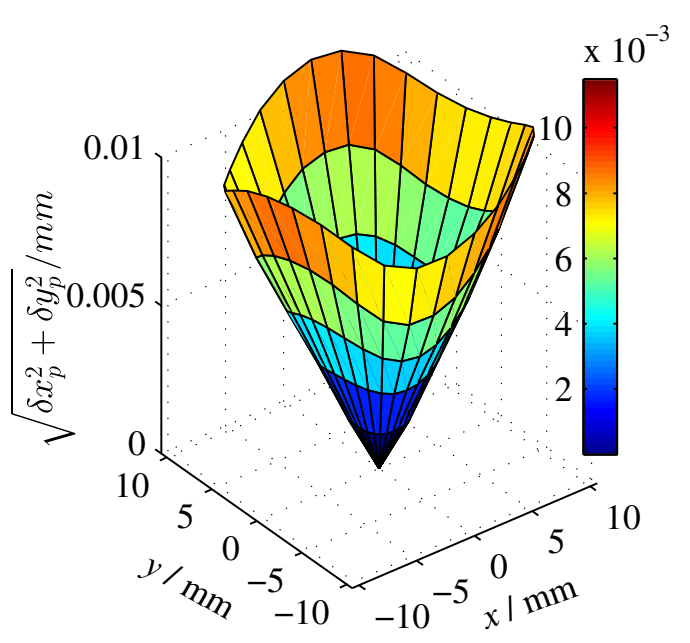

(c)

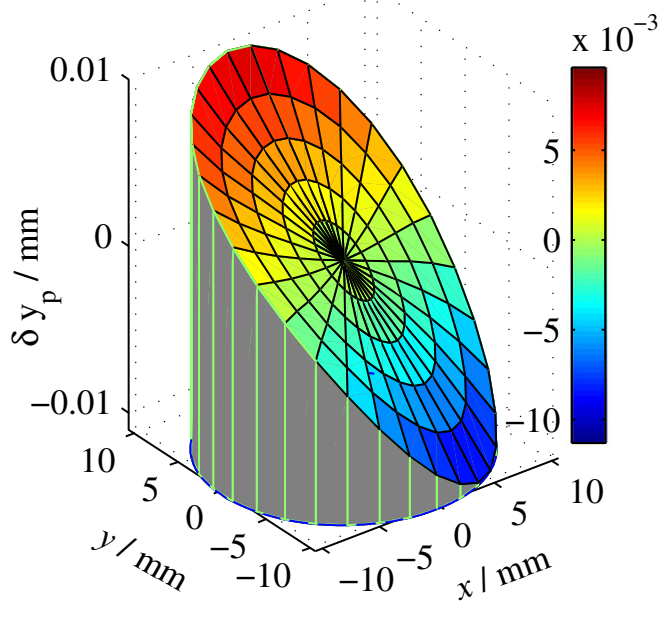

(b)

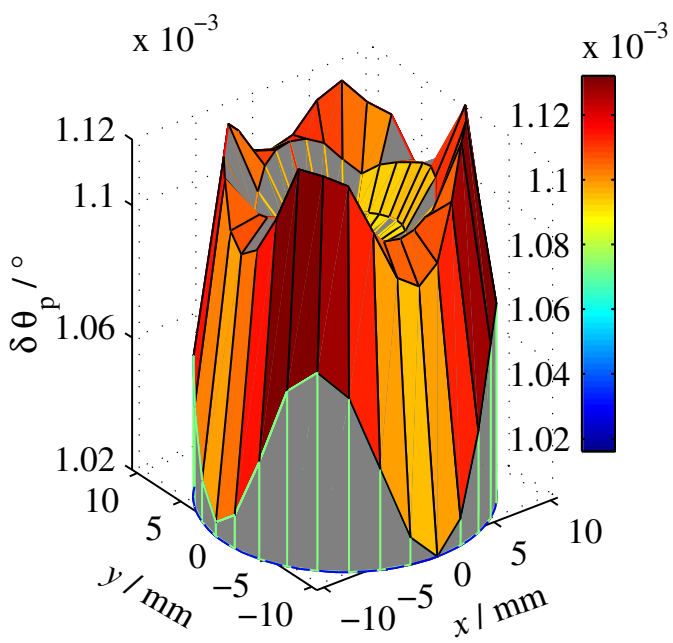

(d)

Fig. 6 Error distribution in whole workspace with $\delta \alpha_{i}=0.01^{\circ}$ only. a Distribution of $\delta x_{\mathrm{p}}$; $\mathbf{b}$ Distribution of $\delta y_{\mathrm{p}} ; \mathbf{c}$ Distribution of $\sqrt{\delta x_{\mathrm{p}}^{2}+\delta y_{\mathrm{p}}^{2}} ; \mathbf{d}$ Distribution of $\delta \theta_{\mathrm{p}}$

From Eqs. 11 and 7, it can be found that $\mathbf{A}=\mathbf{J}_{\mathbf{1}}$, and the elements corresponding to $\delta d_{i}$ from matrix $\mathbf{J}_{2}$ are the same as those of matrix $\mathbf{B}$. This phenomenon shows that the velocity Jacobian matrix also represents the errors transfer relationship, but it only reflects the input errors transfer to the output errors. If one only considers the influence of input errors to output errors, the error transfer matrix in Eq. 7 become dimensionality reduction as the velocity Jacobian matrix.
According to the above relationship, the traditional optimization based on velocity Jacobian matrix like the GCI (Global Condition Index) optimization is not so ideal [18-23], especially for those mechanism whose main error sources are not the actuated joint inputs. Optimization for the parameters of the mechanism only considering the input and output relationship is not enough for the high accurate mechanism.

Using the relationship between the error model and the velocity Jacobian matrix, the generalized 


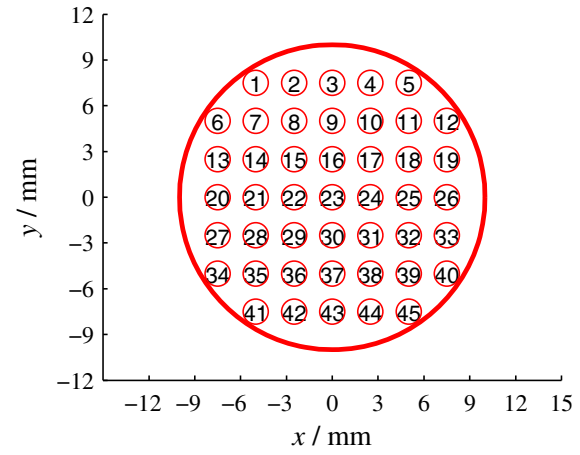

Fig. 7 Circle area mapping with radius $=10 \mathrm{~mm}$, step length $=2.5 \mathrm{~mm}$

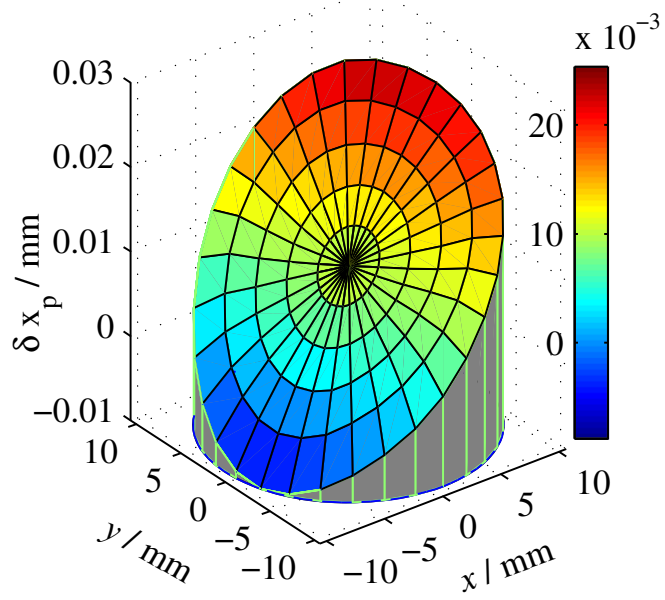

(a)

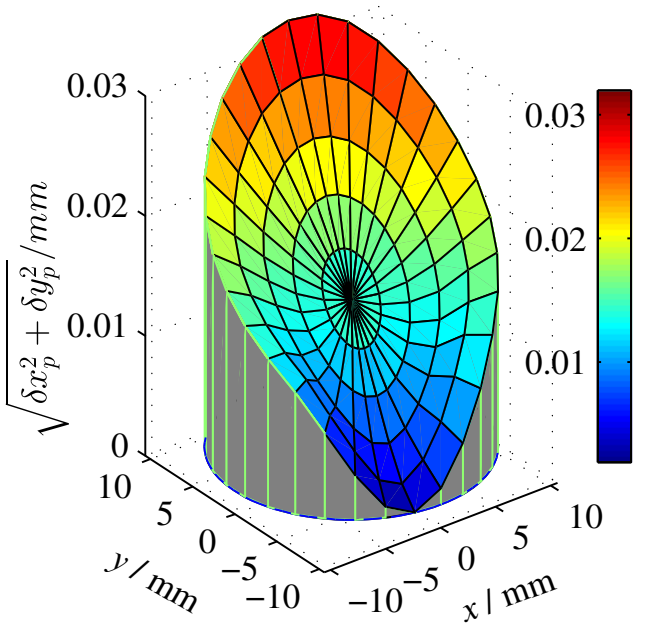

(c)
Jacobian matrix of the $\delta \alpha_{i}(i=1,2,3)$ can be written as

$\delta \mathbf{X}=\mathbf{J}_{\alpha} \cdot \delta \alpha$,

where $\mathbf{J}_{\alpha}=\left[\begin{array}{lll}\cos \theta_{1} & \sin \theta_{1} & \mathrm{~L}_{3-1} \sin \left(\gamma_{1}-\theta_{1}\right) \\ \cos \theta_{2} & \sin \theta_{2} & \mathrm{~L}_{3-2} \sin \left(\gamma_{2}-\theta_{2}\right) \\ \cos \theta_{3} & \sin \theta_{3} & \mathrm{~L}_{3-3} \sin \left(\gamma_{3}-\theta_{3}\right)\end{array}\right]^{-1}$.

$\left[\begin{array}{ccc}-d_{1} \sin \left(\alpha_{1}-\theta_{1}\right) & 0 & 0 \\ 0 & -d_{2} \sin \left(\alpha_{2}-\theta_{2}\right) & 0 \\ 0 & 0 & -d_{3} \sin \left(\alpha_{3}-\theta_{3}\right)\end{array}\right]$;

$\delta \alpha=\left[\begin{array}{lll}\delta \alpha_{1} & \delta \alpha_{2} & \delta \alpha_{3}\end{array}\right]^{\mathrm{T}}$

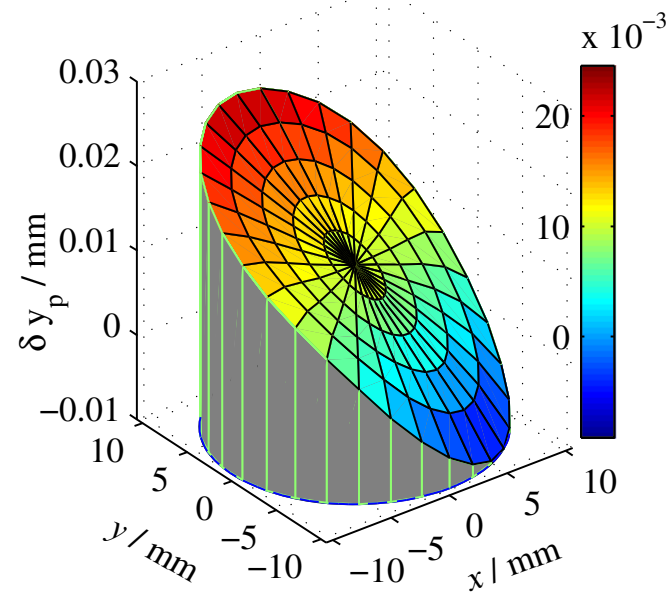

(b)

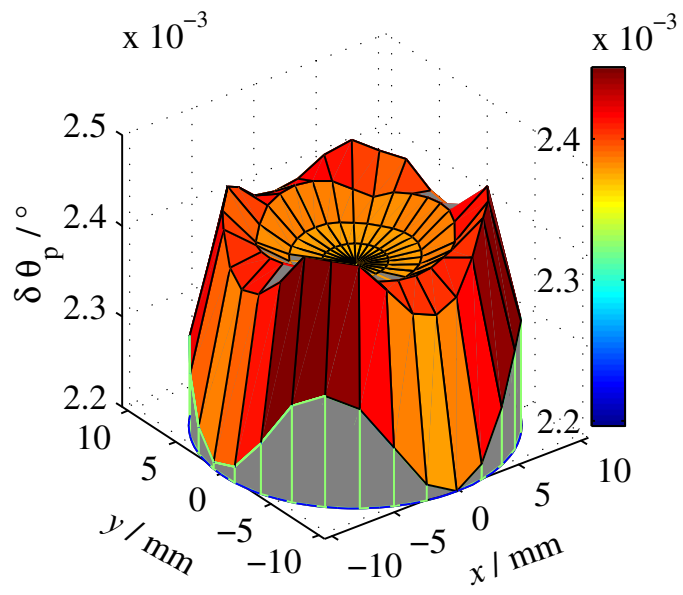

(d)

Fig. 8 Error distribution in whole workspace (Angle errors are all specified as $0.01^{\circ}$; Length errors are all set as $0.01 \mathrm{~mm}$ ). a Distribution of $\delta x_{\mathrm{p}} ; \mathbf{b}$ Distribution of $\delta y_{\mathrm{p}} ; \mathbf{c}$ Distribution of $\sqrt{\delta x_{\mathrm{p}}^{2}+\delta y_{\mathrm{p}}^{2}} ; \mathbf{d}$ Distribution of $\delta \theta_{\mathrm{p}}$ 


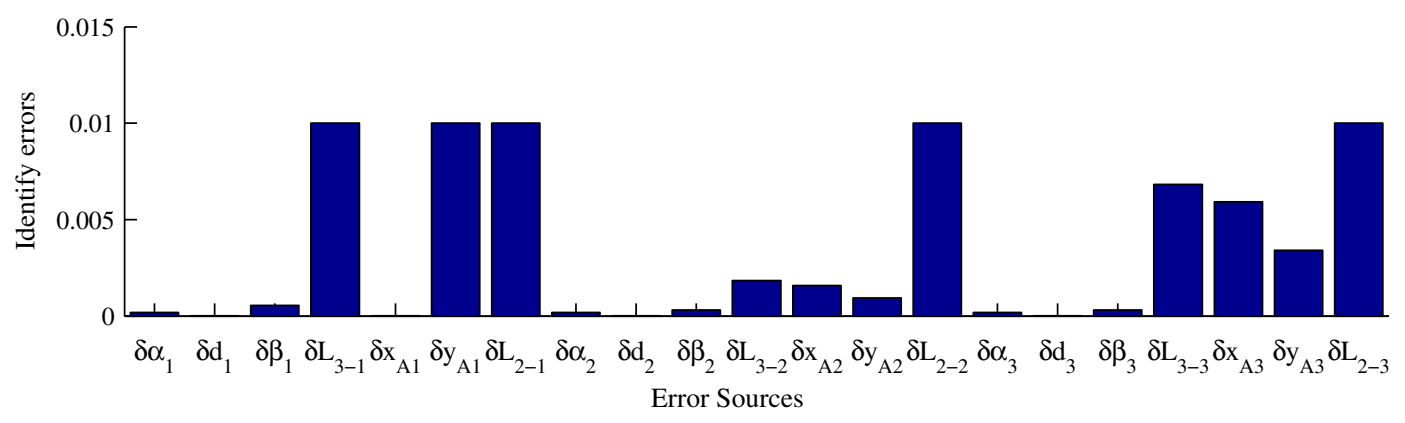

Fig. 9 The identified errors of the simulation

According to Eq. 12, the mapping of the error distribution only with the errors of $\delta \alpha_{i}=0.01^{\circ}$ can be plotted as Fig. 6.

Figure 6 shows the error distribution when the main error source is $\delta \alpha_{i}=0.01^{\circ} . \delta x_{\mathrm{p}}$ and $\delta y_{\mathrm{p}}$ are monotone decreasing. The error $\sqrt{\delta x_{\mathrm{p}}^{2}+\delta y_{\mathrm{p}}^{2}}$ becomes a pyramid shape and the best positioning performance is achieved on the origin point. The distribution of $\delta \theta_{\mathrm{p}}$ is symmetric, and the minimum error is achieved on origin point too. This result conforms to the distribution of the velocity Jacobian matrix of the 3PRR [2]. It demonstrates that the generalized Jacobian matrix is an effective method for error analysis and optimization. The generalized Jacobian matrix can compensate for the disadvantage when optimizing the mechanism with the velocity Jacobian matrix only.

\section{Calibration Method}

The error model can be used as the identified model when the inputs are the $\delta \mathbf{X}$. Using this model, it is able to calibrate the system parameters

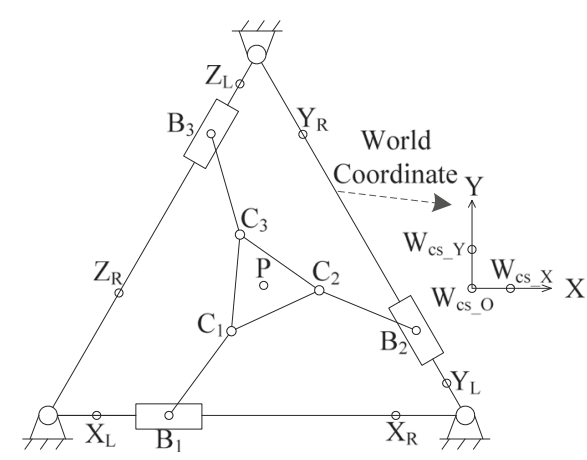

Fig. 10 Assistant measurement points

\subsection{Calibration Based on the Error Model}

According to the above mentioned, the matrix $\mathbf{J}$ is the error transfer matrix of the 3 PRR If the end-effector errors $\delta \mathbf{X}$ are able to be measured then $\delta \mathbf{d}$ can be identified. The error model can be rewritten as the parameters identification model

$\delta \mathbf{d}=\mathbf{J}^{-1} \cdot \delta \mathbf{X}$,

where $\mathbf{J}^{-1}$ is the generalized inverse of $\mathbf{J}$.

Since matrix $\mathbf{J}$ is not a square matrix, and the number of equations is less than the number of unknowns $(3<21)$, Eq. 13 is an indeterminate equation set. In order to identify $\delta \mathbf{d}$ the redundant data of $\delta \mathbf{X}$ are needed. If many positioning points (larger than and equal to 7 points) of the workspace can be measured and so many $\delta \mathbf{X}$ have been obtained, this calibration method based on error model becomes a problem of solving overdetermination equation set.

Assuming that $\mathrm{N}$ points $(\mathrm{N} \geq 7)$ have been measured (13) becomes the over determination equation

Table 3 Calculation of the parameters.

\begin{tabular}{ll}
\hline $\begin{array}{l}\text { Error sources, } \\
(i=1,2,3)\end{array}$ & Description \\
\hline$\alpha_{i}$ & \\
& $\left\langle\mathbf{X}_{\mathbf{L}} \mathbf{X}_{\mathbf{R}}, \mathbf{W}_{\text {cs_o }} \mathbf{W}_{\text {cs_x }}\right\rangle(i=1)$, \\
& $\left\langle\mathbf{Y}_{\mathbf{L}} \mathbf{Y}_{\mathbf{R}}, \mathbf{W}_{\text {cs_o }} \mathbf{W}_{\text {cs_x }}\right\rangle(i=2)$, \\
& $\left\langle\mathbf{Z}_{\mathbf{L}} \mathbf{Z}_{\mathbf{R}}, \mathbf{W}_{\text {cs_o }} \mathbf{W}_{\text {cs_x } \mathbf{x}}\right\rangle(i=3)$ \\
$\mathrm{L}_{2-i}$ & $\left|\mathbf{B}_{\mathbf{i}} \mathbf{C}_{\mathbf{i}}\right|$ \\
$\mathrm{L}_{3-i}$ & $\left|\mathbf{P C}_{\mathbf{i}}\right|$ \\
$\beta_{i}$ & $\left\langle\mathbf{P C}_{\mathbf{i}}, \mathbf{C}_{\mathbf{i}} \mathbf{C}_{\mathbf{i}+\mathbf{1}}\right\rangle$ \\
$\left(x_{\mathrm{A} i} y_{\mathrm{A} i}\right)$ & Intersection of $\mathbf{Z}_{\mathbf{L}} \mathbf{Z}_{\mathbf{R}}$ and $\mathbf{X}_{\mathbf{L}} \mathbf{X}_{\mathbf{R}}(i=1)$ \\
& Intersection of $\mathbf{X}_{\mathbf{L}} \mathbf{X}_{\mathbf{R}}$ and $\mathbf{Y}_{\mathbf{L}} \mathbf{Y}_{\mathbf{R}}(i=2)$ \\
& Intersection of $\mathbf{Y}_{\mathbf{L}} \mathbf{Y}_{\mathbf{R}}$ and $\mathbf{Z}_{\mathbf{L}} \mathbf{Z}_{\mathbf{R}}(i=3)$
\end{tabular}


Table 4 Definitions of the assistant measurement points

\begin{tabular}{|c|c|}
\hline Definitions of the points & Functions \\
\hline $\mathrm{W}_{\text {cso }}$ & The origin point of the world coordinate on the static platform \\
\hline $\mathrm{W}_{\mathrm{csx}}$ & The $\mathrm{x}$-axis point $(20 \mathrm{~mm}$ from the origin $)$ of the world coordinate on the static platform \\
\hline $\mathrm{W}_{\text {csy }}$ & The $y$-axis point $(20 \mathrm{~mm}$ from the origin $)$ of the world coordinate on the static platform \\
\hline $\mathrm{X}_{\mathrm{L}}$ & $\begin{array}{l}\text { Arbitrary point on the left side of the linear guideway } \mathrm{X} \text {, used for fitting a straight line } \\
\text { and measuring } \delta \alpha_{1} \delta x_{\mathrm{A} 1},, \delta y_{\mathrm{A} 1}\end{array}$ \\
\hline $\mathrm{X}_{\mathrm{R}}$ & $\begin{array}{l}\text { Arbitrary point on the right side of the linear guideway } \mathrm{X} \text {, used for fitting a straight line } \\
\text { and measuring } \delta \alpha_{1} \delta x_{\mathrm{A} 1},, \delta y_{\mathrm{A} 1}\end{array}$ \\
\hline $\mathrm{Y}_{\mathrm{L}}$ & $\begin{array}{l}\text { Arbitrary point on the left side of the linear guideway } \mathrm{Y} \text {, used for fitting a straight line } \\
\text { and measuring } \delta \alpha_{2} \delta x_{\mathrm{A} 2} \delta y_{\mathrm{A} 2}\end{array}$ \\
\hline $\mathrm{Y}_{\mathrm{R}}$ & $\begin{array}{l}\text { Arbitrary point on the right side of the linear guideway } \mathrm{Y} \text {, used for fitting a straight line } \\
\text { and measuring } \delta \alpha_{2} \delta x_{\mathrm{A} 2} \delta y_{\mathrm{A} 2}\end{array}$ \\
\hline $\mathrm{Z}_{\mathrm{L}}$ & $\begin{array}{l}\text { Arbitrary point on the left side of the linear guideway } \mathrm{Z} \text {, used for fitting a straight line } \\
\text { and measuring } \delta \alpha_{3} \delta x_{\mathrm{A} 3} \delta y_{\mathrm{A} 3}\end{array}$ \\
\hline $\mathrm{Z}_{\mathrm{R}}$ & $\begin{array}{l}\text { Arbitrary point on the right side of the linear guideway } \mathrm{Z} \text {, used for fitting a straight line } \\
\text { and measuring } \delta \alpha_{3} \delta x_{\mathrm{A} 3} \delta y_{\mathrm{A} 3}\end{array}$ \\
\hline $\mathrm{B}_{1}$ & The endpoint of the linkage on the driving slider used for measuring $\mathrm{L}_{2-1}$ \\
\hline $\mathrm{C}_{1}$ & The endpoint of the linkage on the moving platform used for measuring $\mathrm{L}_{2-1}$ \\
\hline $\mathrm{B}_{2}$ & The endpoint of the linkage on the driving slider used for measuring $L_{2-2}$ \\
\hline $\mathrm{C}_{2}$ & The endpoint of the linkage on the moving platform used for measuring $\mathrm{L}_{2-2}$ \\
\hline $\mathrm{B}_{3}$ & The endpoint of the linkage on the driving slider used for measuring $\mathrm{L}_{2-3}$ \\
\hline $\mathrm{C}_{3}$ & The endpoint of the linkage on the moving platform used for measuring $\mathrm{L}_{2-3}$ \\
\hline $\mathrm{P}$ & The end-effector point used for measuring $\mathrm{L}_{3-1} \mathrm{~L}_{3-2}, \mathrm{~L}_{3-3} \delta \beta_{1}, \delta \beta_{2} \delta \beta_{3}$ \\
\hline
\end{tabular}

set. Considering each chain respectively, it can be rewritten as dimensionality reduction form of

$$
\left[\begin{array}{c}
\mathbf{A}_{i i_{-} 1} \\
\mathbf{A}_{i i \_2} \\
\vdots \\
\mathbf{A}_{i i_{-} N}
\end{array}\right]_{N \times 7}^{-1}\left[\begin{array}{c}
\mathbf{J}_{1 \_\mathrm{R}(i) \_1} \delta \mathbf{X}_{1} \\
\mathbf{J}_{1 \_\mathrm{R}(i) \_2} \delta \mathbf{X}_{2} \\
\vdots \\
\mathbf{J}_{1 \_\mathrm{R}(i) \_N} \delta \mathbf{X}_{N}
\end{array}\right]_{N \times 1}=\delta \mathbf{d}_{i}
$$

where $\delta \mathbf{X}_{1}, \ldots, \delta \mathbf{X}_{N}$ are the measured end-effector errors; $\mathbf{J}_{1 \_\mathrm{R}(i)}$ represent the $i$ th row of $\mathbf{J}_{1},(i=1,2,3)$.

Based on the principle of pseudo-inverse of matrix, Eq. 14 can be used to calculate the least squares solution of $\delta \mathbf{d}_{i}(i=1,2,3)$ then the error sources of each chain can be identified.

For a calculation example, assuming the angle errors are all specified as $0.01^{\circ}$ and the length errors are all $0.01 \mathrm{~mm}$ The other parameters are described previously. Simulation of the errors distribution with equidistant points is carried out. The points mapping scheme is defined in Fig. 7.

Substituting $\delta \mathbf{d}$ into the error model (7), and calculating the errors of the end-effector at each point of Fig. 7, the error distribution in whole workspace is obtained and shown in Fig. 8.

Table 5 The assistant measurement points of simulation

\begin{tabular}{llllllllllllllll}
\hline Points & $\mathrm{B}_{1}$ & $\mathrm{~B}_{2}$ & $\mathrm{~B}_{3}$ & $\mathrm{C}_{1}$ & $\mathrm{C}_{2}$ & $\mathrm{C}_{3}$ & $\mathrm{O}$ & $\mathrm{P}$ & $\mathrm{X}_{\mathrm{L}}$ & $\mathrm{X}_{\mathrm{R}}$ & $\mathrm{Y}_{\mathrm{L}}$ & $\mathrm{Y}_{\mathrm{R}}$ & $\mathrm{Z}_{\mathrm{L}}$ & $\mathrm{Z}_{\mathrm{R}}$ & \\
\hline $\mathrm{X} / \mathrm{mm}$ & -40.39 & 114.54 & -60.64 & 4.03 & 20.43 & 27.29 & 0.00 & -0.94 & -181.86 & 181.86 & 181.86 & 0.00 & 0.00 & -181.86 \\
$\mathrm{Y} / \mathrm{mm}$ & -105.00 & 11.60 & 104.95 & -21.02 & 24.61 & 16.00 & 0.00 & 6.53 & -105.00 & -105.00 & -105.00 & 210.00 & 210.00 & -105.00
\end{tabular}


Table 6 The simulation results of the assistant measurement

\begin{tabular}{llll}
\hline Parameters $(i=\mathrm{X}$ or $\mathrm{Y}$ or $\mathrm{Z})$ & Joint $\mathrm{X}$ & Joint $\mathrm{Y}$ & Joint Z \\
\hline$\alpha_{i} /{ }^{\circ}$ & 0.00 & 120.00 & 240.00 \\
$d_{i} / \mathrm{mm}$ & 131.81 & 131.81 & 131.81 \\
$\beta_{i} /{ }^{\circ}$ & 29.99 & 30.00 & 30.00 \\
$\mathrm{~L}_{3-i} / \mathrm{mm}$ & 28.00 & 28.00 & 28.00 \\
$x_{\mathrm{A} i} / \mathrm{mm}$ & -181.86 & 181.86 & 0.00 \\
$y_{\mathrm{A} i} / \mathrm{mm}$ & -105.00 & -105.00 & 210.00 \\
$\mathrm{~L}_{2-i} / \mathrm{mm}$ & 94.99 & 95.00 & 95.00 \\
\hline
\end{tabular}

Figure 8 shows the errors distribution of this $3 \underline{P R R}$ positioning system in whole workspace. The distributions of $\delta x_{\mathrm{p}}$ and $\delta y_{\mathrm{p}}$ are not symmetrical and presenting the monotone decreasing feature. The tendency of the errors $\sqrt{\delta x_{\mathrm{p}}^{2}+\delta y_{\mathrm{p}}^{2}}$ is the same as that of $\delta x_{\mathrm{p}}$ and $\delta y_{\mathrm{p}}$. The distribution of $\delta \theta_{\mathrm{p}}$ is symmetrical and the error is minimum in original point. At the original point, there are about $0.01 \mathrm{~mm}$ positioning error in both $\delta x_{\mathrm{p}}$ and $\delta y_{\mathrm{p}}$. Comparing with the Figs. 6 and 8 , the tendency of the error mappings $\left(\delta x_{\mathrm{p}} \delta y_{\mathrm{p}}, \delta \theta_{\mathrm{p}}\right)$ are all consistent. But the value in Fig. 8 is bigger than that in Fig. 6, because more errors are introduced in Figs. 8 than Fig. 6. The $\delta \alpha_{i}$ are the main error sources, and influence the end-effector errors greatly. It can be found that the range of $\delta x_{\mathrm{p}}$ and $\delta y_{\mathrm{p}}$ in Fig. 8 is about $-0.01 \mathrm{~mm}$ to $0.03 \mathrm{~mm}$ If only introduce $\delta \alpha_{i}=0.01^{\circ}$ into the system, the range of $\delta x_{\mathrm{p}}$ and $\delta y_{\mathrm{p}}$ in Fig. 6 become $\mathrm{s}-0.01 \mathrm{~mm}$ to $0.01 \mathrm{~mm}$, it is account for a large part of the end-effector errors, which proving that $\delta \alpha_{i}$ is the main error sources. In Figs. 6 and 8, the

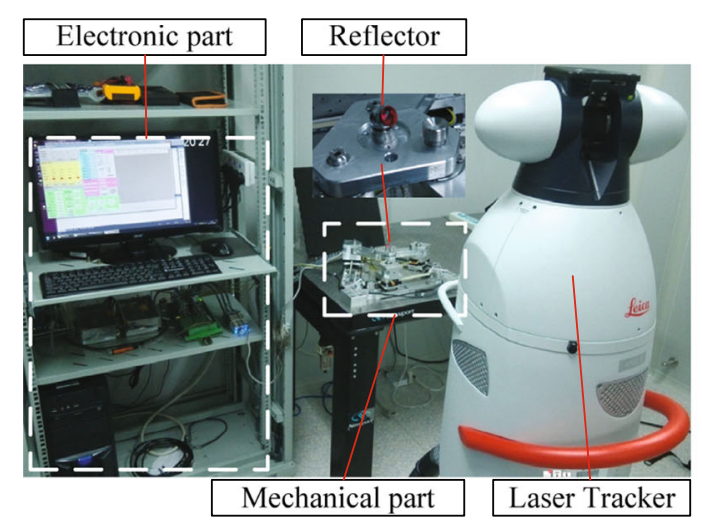

(a) Every part of the experimental system;

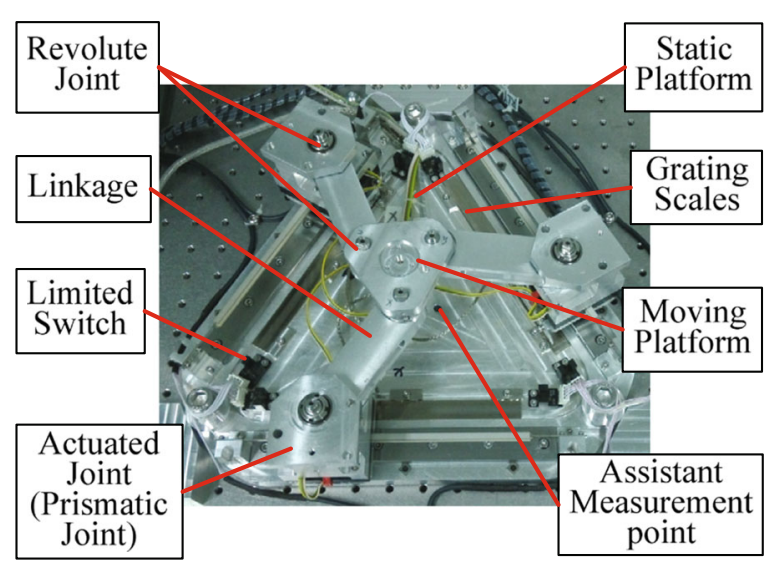

Fig. 12 Construction of $3 \underline{P} R R$ parallel mechanism

tendency of the error $\sqrt{\delta x_{\mathrm{p}}^{2}+\delta y_{\mathrm{p}}^{2}}$ mappings is consistent too. But the minimum point in Fig. 6 is near the origin point, while the minimum point in Fig. 8 translates for a distance, and the mapping outside the workspace is not being plot in Fig. 8 .

In order to verify the identify model, substituting $\delta x_{\mathrm{p}} \delta y_{\mathrm{p}}$ and $\delta \theta_{\mathrm{p}}$ back into Eq. 14 , the calculation results are shown as Fig. 9.

Figure 9 shows the identified results of 21 error sources. The results show that some of the parameters values are near 0.01 , which verify the assumption, but some of the parameters values are near 0 , which means that these parameters are difficult to be identified by using the decoupling error model. The reason why this phenomenon happens is dependent on the model structure of the identified model. The simplified model is irreversible and the exact solution cannot

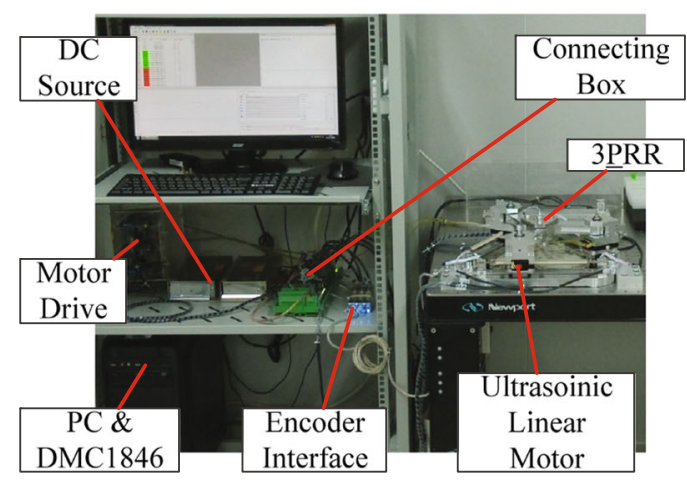

(b) The 3PRR positioning system;

Fig. 11 Photographs of the experimental setups 


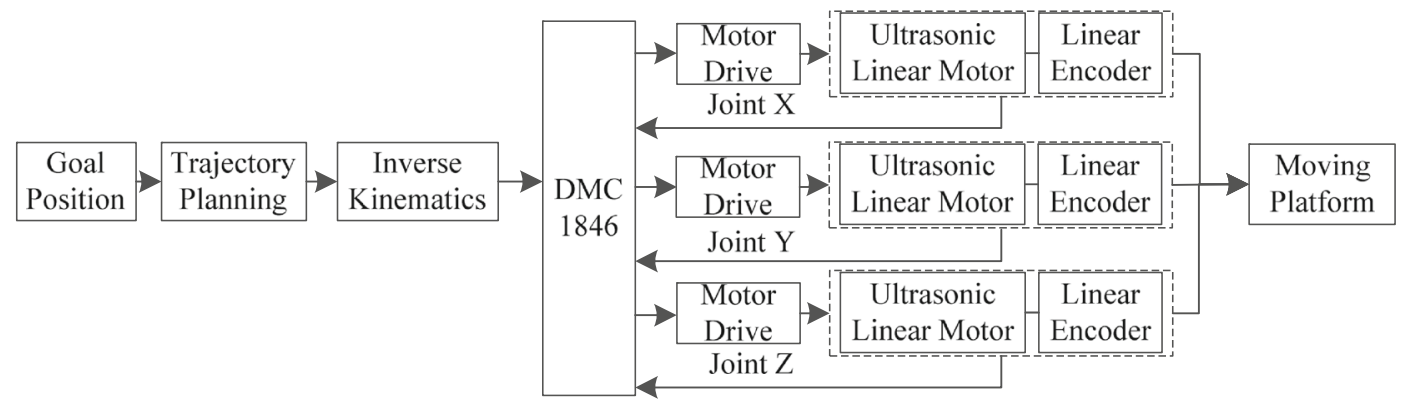

Fig. 13 The block diagram of the $3 \underline{P R R}$ positioning system

be obtained by using the overdetermined equation set, which limited the accuracy of identification.

According to the GESI distribution, the most sensitive error sources are $\delta \alpha_{i}(i=1,2,3)$. However, the simulation results of the identification show that $\delta \alpha_{i}(i=1,2,3)$ is identified inaccurately. In spite of the other errors are identified accurately, the calibration results are not ideal, and the other assistant measurements are needed.

\subsection{The Assistant Measurements for Calibration}

Based on the error model, there are 21 error sources. Some of the error sources are easy to be identified by utilizing the error model, others are difficult. Thus, the assistant measurement is needed to improve the calibration. In the designing process, some of the points using for calibration are designed, corresponding to the 21 error sources. The assistant measurement points are shown as Fig. 10.

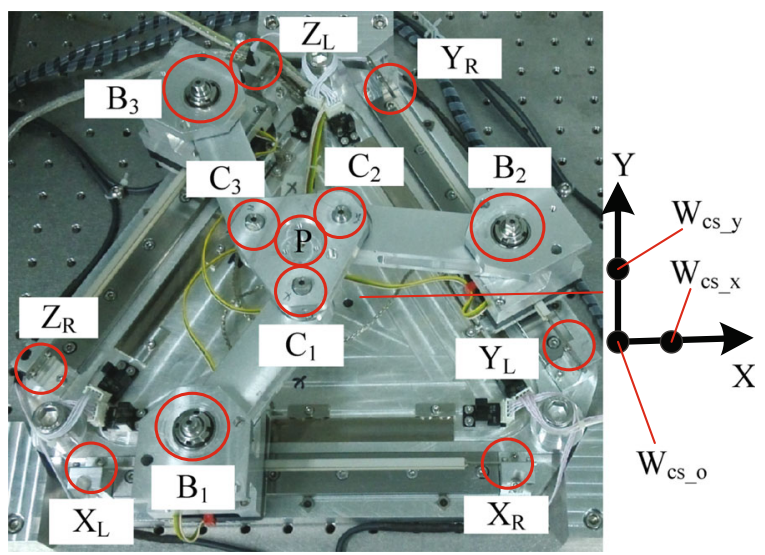

Fig. 14 The design of the assistant measurement points
Figure 10 shows 16 assistant measurement points applied to calibrate the system. Three joints have been defined as joint $\mathrm{X}$, joint $\mathrm{Y}$, and joint $\mathrm{Z}$ around the origin point in counter-clockwise direction (Table 3 ). The functions of these points are list in Table 4.

In the design process, there are three points named $\mathrm{W}_{\text {cs_o }}, \mathrm{W}_{\text {cs_x }}$ and $\mathrm{W}_{\text {cs_y }}$ locating on the static platform used for creating the reference coordinate system, and the subsequent measurements are based on it. The measurement points defined in Table 4 are able to compensate for uncertainty from the identified model. If these points are able to be measured, the geometrical relationship like the length and the angle of the system parameters are easy to be calculated, and the equations are listed in Table 3. The symbol $\langle\mathbf{A}, \mathbf{B}\rangle$ represents the angle between vectors $\mathbf{A}$ and $\mathbf{B}$. The symbol $|\mathbf{A}|$ represents the norm of vectorA.

The simulation measurement points are list in Table 5, they are corresponding to the assistant measurement points in Fig. 10. Calculating the points listed in Table 5, the parameters of the 3PRR are obtained and listed in Table 6. The results show that the assistant measurement can identify the parameters while the error model cannot. However, the assistant measurements will introduce the measuring error, like the manufacturing errors. Although these errors

Table 7 The measured value of the main error sources

\begin{tabular}{lllllll}
\hline Values $^{\circ}$ & $\alpha_{1}$ & $\alpha_{2}$ & $\alpha_{3}$ & $\beta_{1}$ & $\beta_{2}$ & $\beta_{3}$ \\
\hline Theoretical & 360.00 & 120.00 & 240.00 & 30.00 & 30.00 & 30.00 \\
Measure 1 & 360.02 & 119.97 & 239.92 & 30.27 & 30.05 & 29.68 \\
Measure 2 & 359.90 & 119.91 & 239.96 & 29.96 & 30.16 & 29.88 \\
Measure 3 & 359.98 & 119.95 & 239.97 & 29.96 & 30.13 & 29.91 \\
Measure 4 & 359.98 & 119.98 & 239.99 & 30.29 & 30.00 & 29.71 \\
Average & 359.95 & 119.95 & 239.96 & 30.12 & 30.08 & 29.79
\end{tabular}


affect the absolute position measurement, they do not affect the relative positioning measurement. Like the fitting of the linear guideway, $\alpha_{i}(i=1,2,3)$ can be identified accurately.

\section{Experiments}

Experiments are conducted to verify the calibration method with assistant measurement. The $3 \underline{P R R}$ positioning system driven by ultrasonic linear motors is constructed. Experimental results of the calibration method are presented

\subsection{Experimental System Description}

The experimental apparatus of the experimental setup is depicted in Fig. 11a. The ultrasonic linear

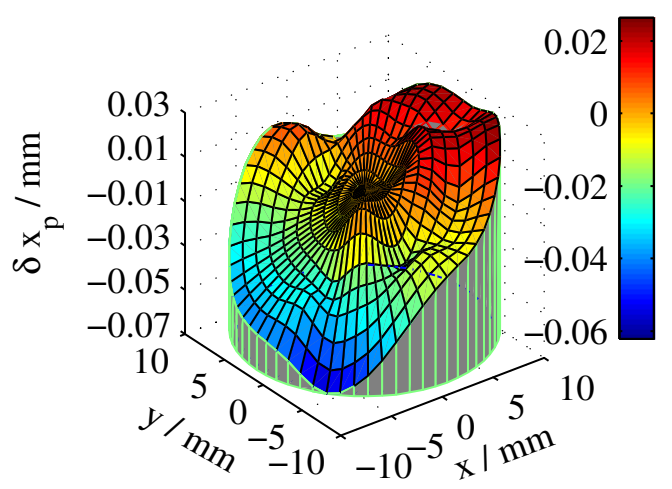

(a)

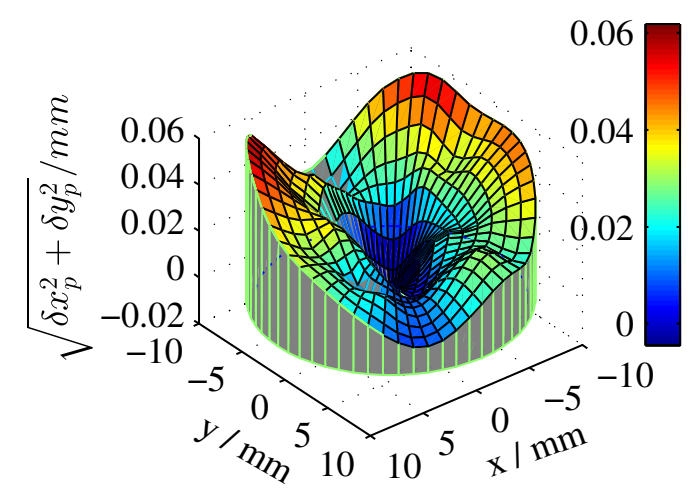

(c) motor driving $3 \underline{P} R R$ positioning system is shown in Fig. 11b. The calibration of this experimental apparatus includes the measurement part and the $3 \underline{P R R}$ positioning system. The Leica laser tracker measurement system (AT901-B) is used and it has capability to measure the position of the single point in Cartesian coordinate with the $0.01 \mu \mathrm{m}$ resolution and $10 \mu \mathrm{m}$ precision, with the tracking length up to $50 \mathrm{~m}$. The AT901-B measurement system can satisfy the measurement requirements. The 3 PRR positioning system includes the mechanical part and the electronic part. The mechanical part of the 3 PRR positioning system has three uniform branched chains (Fig. 12). Each chain is composed of the motor stands, linear slider and the ultrasonic linear motor. The moving platform is connected to three chains with three linkages. The electronic part of the system comprises three linear encoders six limit switches,

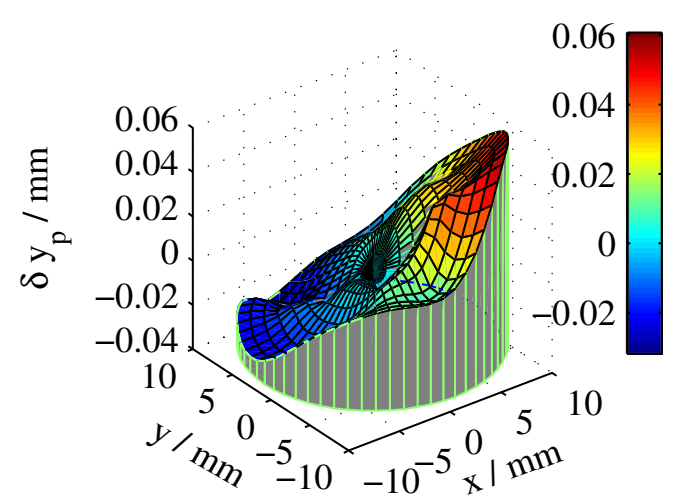

(b)

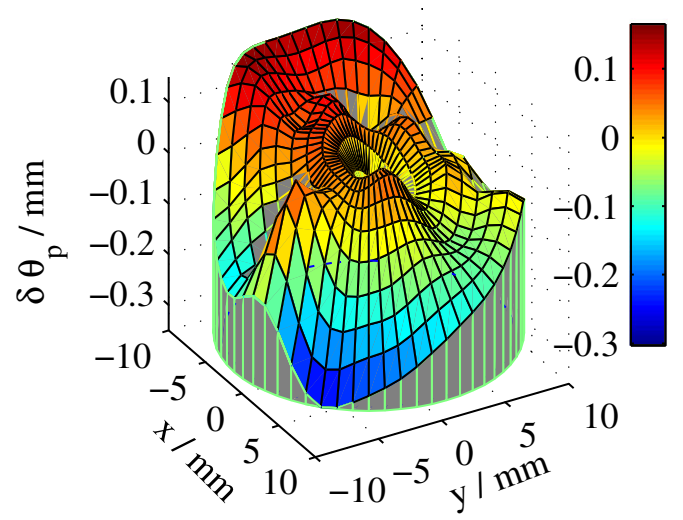

(d)

Fig. 15 The measurement error distributions using the designed parameters. a Distribution of $\delta x_{\mathrm{p}} ; \mathbf{b}$ Distribution of $\delta y_{\mathrm{p}} ; \mathbf{c}$ Distribution of $\sqrt{\delta x_{\mathrm{p}}^{2}+\delta y_{\mathrm{p}}^{2}} ; \mathbf{d}$ Distribution of $\delta \theta_{\mathrm{p}}$ 
three motor drive units, a PC and the motion control card.

The 3PRR positioning system is a semi closedlooped system with the actuated joint feedback only. The PI U-264 ultrasonic linear motor receives the ultrasonic drive voltage from the PI C-872 motor drive unit. Then, the motor will move in a specific velocity carrying the Renishaw Tonic linear encoder (resolution $0.05 \mu \mathrm{m}$ ). The encoder can measure the actual displacement and velocity used as feedback signals. The feedback signals are sent to the incremental encoder interface of the Galil DMC1846 motion control card. The DMC1846 motion control card receives the displacement signal and sends it to the selfdesigned secondary development software interface which is programmed by C\# language. The control command value is calculated through the specific algorithm, and the command value will be converted to an analog voltage and sent through the D/A converter interface $(16$ bits, $-10 \mathrm{~V} \sim+10 \mathrm{~V} \mathrm{DC})$ of DMC1846 to the motor drive unit. The motor drive unit will amplify and convert the DC reference signal to the high frequency $(160 \mathrm{kHz})$, high amplitude (up to 200V) AC drive voltage to the PZT inside the motor case. Thus, the motor adjusts the displacement and velocity in real time. The Omron photoelectric switches are used as the limit switch. The flow chart of the signals is shown in Fig. 13.

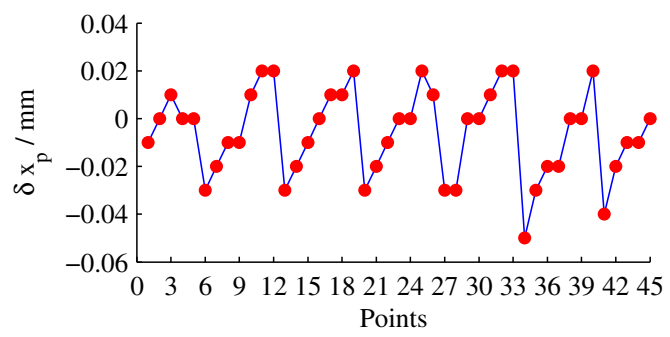

(a)

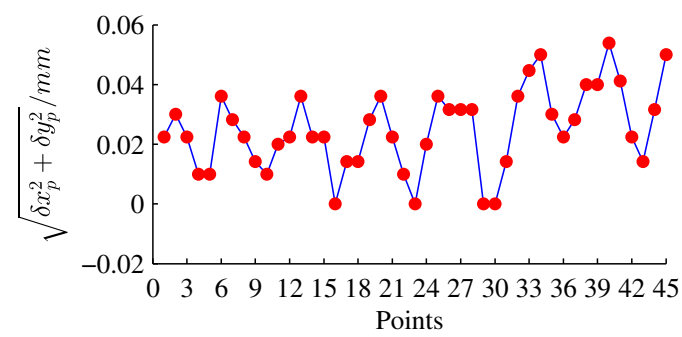

(c)
Table 8 Identified parameters

\begin{tabular}{llll}
\hline & Chain 1 & Chain 2 & Chain 3 \\
\hline$\alpha_{i} / \mathrm{rad}$ & 0.00134 & 2.09692 & 4.18866 \\
$d_{i} / \mathrm{mm}$ & 0.00232 & -0.00026 & 0.00095 \\
$\beta_{i} / \mathrm{rad}$ & 0.45858 & 2.62522 & 4.68567 \\
$\mathrm{~L}_{3-\mathrm{i}} / \mathrm{mm}$ & 29.06804 & 27.84005 & 28.29452 \\
$x_{\mathrm{A} i} / \mathrm{mm}$ & -181.86300 & 182.00400 & 0.25459 \\
$y_{\mathrm{A} i} / \mathrm{mm}$ & -103.93200 & -104.92000 & 209.85190 \\
$\mathrm{~L}_{2-\mathrm{i}} / \mathrm{mm}$ & 92.07273 & 95.12092 & 94.09782 \\
\hline
\end{tabular}

The whole positioning system has three uniform actuated joints. When the system begins to move, the desired goal position of the workspace is converted into the targeted joints position via the inverse kinematics equation. If the parameters of the 3 PRR system can be identified accurately, the positioning of the system will be precision.

\subsection{Calibration experiments}

The calibration experiments are based on the identification. The experimental procedure is described as: Positioning the end-effector with the designed parameters; measuring the end-effector errors; identifying the parameters through the identified model; and then the parameters of the system can be calculated. Using

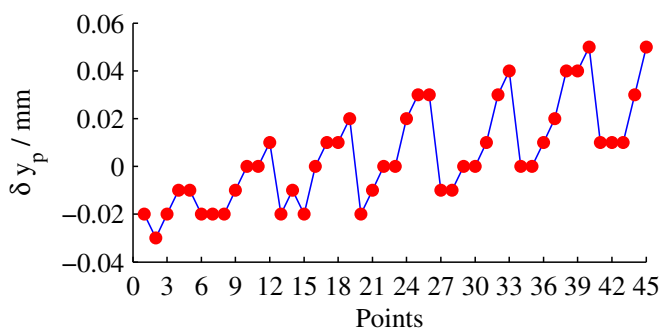

(b)

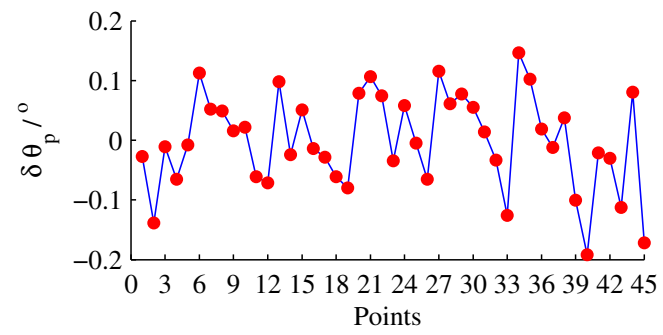

(d)

Fig. 16 The measurement error curves using the design parameters. a $\delta x_{\mathrm{p}} ; \mathbf{b} \delta y_{\mathrm{p}} ; \mathbf{c} \sqrt{\delta x_{\mathrm{p}}^{2}+\delta y_{\mathrm{p}}^{2}} ; \mathbf{d} \delta \theta_{\mathrm{p}}$ 
the identified parameters to calibrate the system the positioning accuracy and errors of the system can be obtained.

Since the identified model cannot be used to identify the main error sources, the main error sources are obtained through the assistant measurement. The design of the assistant measurement points is shown in Fig. 14. Using the Leica laser tracker to measure these points, the values of $\alpha_{i}(i=1,2,3)$ and $\beta_{i}(i=$ $1,2,3)$ can be calculated and shown in Table 7.

The values of $\alpha_{i}(i=1,2,3)$ and $\beta_{i}(i=1,2,3)$ are used to compensate for the unidentified values by the identified model. Before calibration, the error distributions are measured by utilizing the laser tracker (shown in Fig. 15), using the points mapping plan illustrated in Fig. 7.

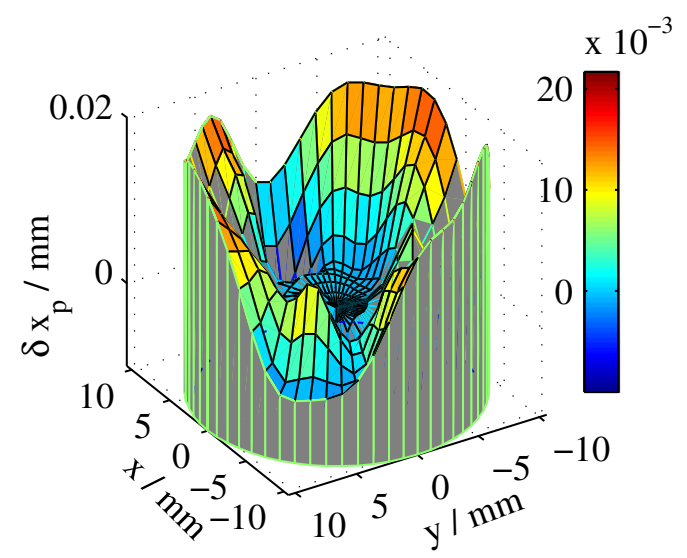

(a)

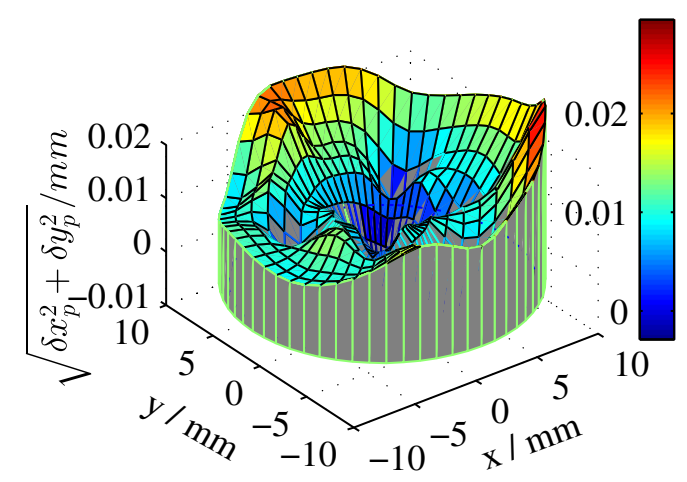

(c)
Figure 15 shows that the measurement error distributions are similar with the simulation results of Fig. 8. It is demonstrated that the error model conforms to the actual performance. Plotted the errors curves in Fig. 16, one knows that the positioning error $\delta x_{\mathrm{p}}$ and $\delta y_{\mathrm{p}}$ are up to $0.05 \mathrm{~mm}$, and the orientation error $\delta \theta_{\mathrm{p}}$ is up to $0.05^{\circ}$. Substituting the measurement errors into the identified model, the identified parameters are obtained and list in Table 8.

Table 8 shows the identified parameters calculated by the identified model. The $\alpha_{i}(i=1,2,3)$ and $\beta_{i}(i=1,2,3)$ are identified not so accurate. Therefore, the angles $\alpha_{i}(i=1,2,3)$ and $\beta_{i}(i=1,2,3)$ in Table 8 are being replace with the assistant measurement value in Table 8 . So the system parameters have been calibrated Then, the calibrated parameters

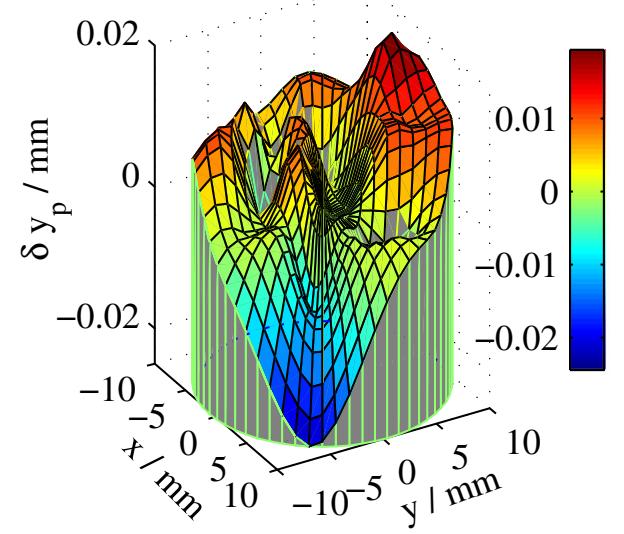

(b)

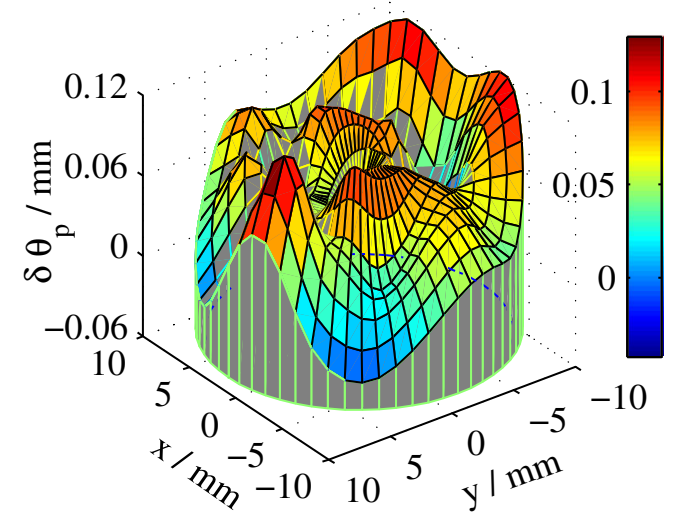

(d)

Fig. 17 The measurement error distributions using the calibrated parameters. a Distribution of $\delta x_{\mathrm{p}}$; b Distribution of $\delta y_{\mathrm{p}}$; c Distribution of $\sqrt{\delta x_{\mathrm{p}}^{2}+\delta y_{\mathrm{p}}^{2}} ; \mathbf{d}$ Distribution of $\delta \theta_{\mathrm{p}}$ 


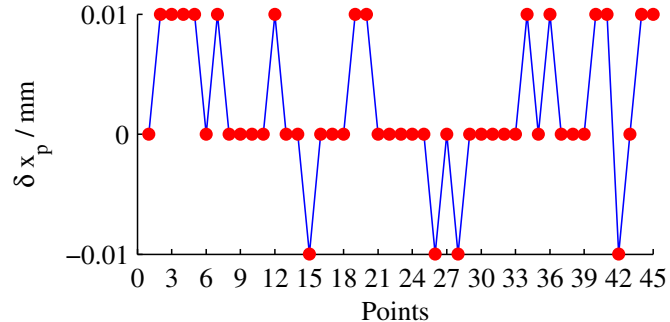

(a)

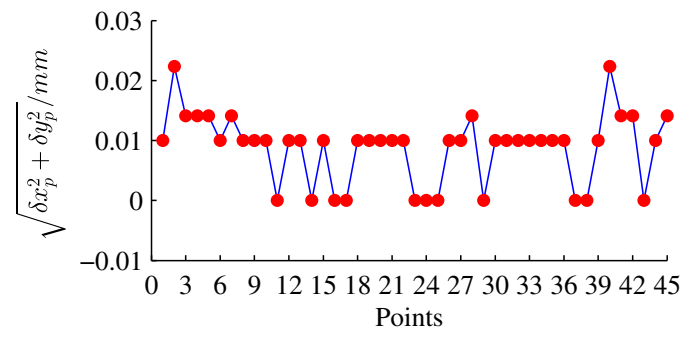

(c)

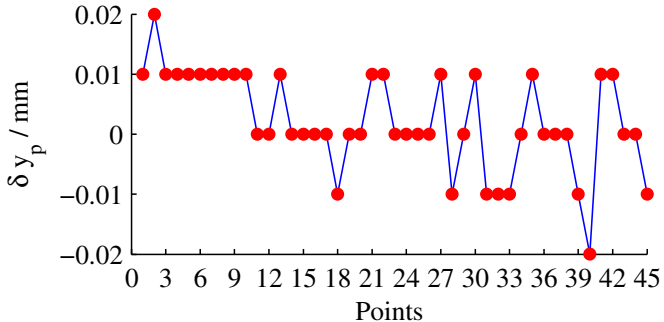

(b)

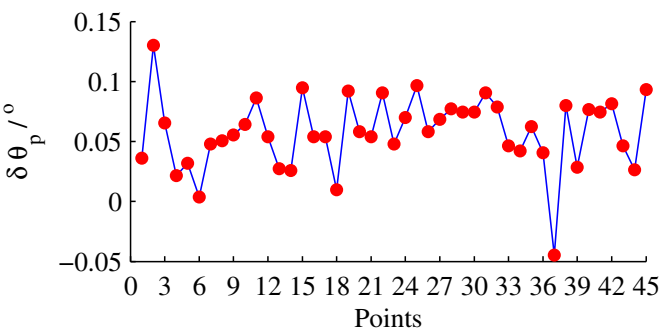

(d)

Fig. 18 The measurement error curves using the calibrated parameters. a $\delta x_{\mathrm{p}} ; \mathbf{b} \delta y_{\mathrm{p}} ; \mathbf{c} \sqrt{\delta x_{\mathrm{p}}{ }^{2}+\delta y_{\mathrm{p}}^{2}} ; \mathbf{d} \delta \theta_{\mathrm{p}}$

are being applied to the system. The error distributions are plotted in Fig. 17, and the curve are plotted in Fig. 18.

The experimental results shows that the positioning errors reduce to about $\pm 10 \mu \mathrm{m}$ and the orientation errors reduce to about $0.05^{\circ}$ after calibration. Because the accuracy degree of the AT901-B laser tracker is $10 \mu \mathrm{m}$, the measurement data displayed zero not means the errors is zero actually. In fact it means the absolute errors are less than $\pm 5 \mu \mathrm{m}$. After calibration, the errors distributions become a bowl shape, and appear the better positioning performance near the origin point. This phenomenon conforms to the properties of the parallel mechanism. The end-effector will be near the singular boundary of the workspace when it is far away from the origin point, and the error transfer will become more and larger when the end-effector is near the singular boundary.

\section{Conclusions}

This paper proposes a new calibration method for a 3PRR parallel manipulator. Firstly, a kind of directly driven 3PRR positioning system is presented and constructed. The error modeling and error analysis of the 3PRR positioning system are investigated. After analyzing the errors, it can be found that the angle errors of the guideway are the main error sources in this 3 PRR positioning system. The parameters identification model has also been derived. The simulation results demonstrate that it is not so ideal for parameters identification, because some parameters are unable to be identified accurately via the general calibration method. Therefore, the assistant measurement method is proposed to compensate for this disadvantage. The experiment results demonstrate that this new calibration method based on the assistant measurement can exert satisfactory positioning effect, and meet the requirements of micron grade positioning. Furthermore, the calibration accuracy is relative to the measurement accuracy.

Acknowledgments This work was supported by the National Natural Science Foundation of China (Grant Nos. U1501247, 91223201), the Natural Science Foundation of Guangdong Province (Grant No. S2013030013355) the Scientific and Technological Project of Guangzhou (Grant No.2015090330001) and the Science and Technology Planning Project of Guangdong Province (Grant No. 2014B090922001). The authors gratefully acknowledge these support agencies.

Open Access This article is distributed under the terms of the Creative Commons Attribution 4.0 International License (http://creativecommons.org/licenses/by/4.0/), which permits unrestricted use, distribution, and reproduction in any medium, provided you give appropriate credit to the original author(s) and the source, provide a link to the Creative Commons license, and indicate if changes were made. 


\section{References}

1. Merlet, J.P.: Parallel Robot (Second Edition)[M], pp. 2729. Springer Science \& Business Media, Dordrecht (2012)

2. Mo, J., Zhang, X., Qiu, Z., et al.: Control strategy research on avoid and escape the singular area of the 3PRR parallel platform based on SEM environment [J]. J. Mech. Eng. 51(23), 1-11 (2015)

3. Tsai, L.: Robot Analysis: The Mechanics of Serial and Parallel Manipulators [M]. Wiley (1999)

4. Mo, J., Qiu, Z., Wei, J., et al.: Experimental study on joint positioning control of an ultrasonic linear motor driven planar parallel platform [J]. Intelligent robotics and applications, pp. 48-59. Springer International Publishing (2014)

5. Spong, M.W., Vidyasagar, M.: Robot Dynamics and Control [M]. Wiley (2008)

6. Chen, W.H.: Disturbance observer based control for nonlinear systems [J]. IEEE/ASME Trans. Mechatron. 9(4), 706-710 (2004)

7. Saha, S.K.: Introduction to Robotics [M]. Tata McGrawHill Education (2014)

8. Zhou, N., Hao, K., Guo, C., et al.: Visual Servo Control System of 2-DOF Parallel Robot [Z], pp. 425-433. Springer (2012)

9. Pradeep, V., Konolige, K., Berger, E.: Calibrating a MultiArm Multi-Sensor Robot: a Bundle Adjustment Approach [C]. Springer (2014)

10. Shao, Z., Tang, X., Wang, L., et al.: Self-Calibration Method of planar flexible 3-RRR parallel manipulator [J]. Chinese J. Mech. Eng. 45(3), 150-155 (2009)

11. Yang, Q., Sun, Z., Yan, M., et al.: Kinematic reliability of improved delta parallel mechanism [J]. Acta Aeronautica Et Astronautica Sinica 02, 487-491 (2008)

12. Zi Xing, C.: Robotics [M]. Tsinghua University Press, Tsinghua (2000)

13. Nubiola, A., Bonev, I.A.: Absolute robot calibration with a single telescoping ballbar [J]. Precis. Eng. 38(3), 472-480 (2014)

14. Jiménez, P.A., Shirinzadeh, B.: Laser interferometry measurements based calibration and error propagation identification for pose estimation in mobile robots [J]. Robotica 32(01), 165-174 (2014)

15. Xin, X., Shen, H., Wang, P.: Kinematics calibration of a 3-DOF parallel mechanism [J]. Machinery Design \& Manufacture 7, 38-40 (2007)

16. Nubiola, A., Slamani, M., Joubair, A., et al.: Comparison of two calibration methods for a small industrial robot based on an optical c MM and a laser tracker [J]. Robotica 32(03), 447-466 (2014)

17. Wang, W., Liu, F., Yun, C.: Calibration method of robot base frame using unit quaternion form [J]. Precis. Eng. 41, 47-54 (2015)
18. Caro, S., Chablat, D., Ur-Rehman, R., et al.: Multiobjective Design Optimization of 3-PRR Planar Parallel Manipulators [Z], pp. 373-383. Springer (2011)

19. Gosselin, C., Angeles, J.: The optimum kinematic design of a planar three-degree-of-freedom parallel manipulator [J]. J. Mech. Des. 110(1), 35-41 (1988)

20. Gosselin, C., Angeles, J.: A global performance index for the kinematic optimization of robotic manipulators $[\mathrm{J}]$. J. Mech. Des. 113(3), 220-226 (1991)

21. Zhu, Z., Dou, R.: Optimum design of 2-DOF parallel manipulators with actuation redundancy $[\mathrm{J}]$. Mechatronics 19(5), 761-766 (2009)

22. Liu, X., Jin, Z., Gao, F.: Optimum design of 3-DOF spherical parallel manipulators with respect to the conditioning and stiffness indices [J]. Mech. Mach. Theory 35(9), 12571267 (2000)

23. Gao, F., Liu, X., Gruver, W.: The Global Conditioning Index in the Solution Space of Two Degree of Freedom Planar Parallel Manipulators [C]. IEEE (1995)

Jiasi Mo was born in Guangzhou, Guangdong, China, in 1987. He received the Ph.D. degree from South China University of Technology, Guangzhou, China, in 2016. He is currently working in South China Agricultural University. His research interests include parallel mechanism control, agricultural robot and deep-sea aquaculture mechanism.

Zhicheng Qiu was born in 1973. He received the Ph.D. degree from the Harbin Institute of Technology, Harbin, China, in 2000. He has been a Professor with the South China University of Technology, Guangzhou, China, since 2009. His research interests include Modeling and control of flexible structure, robotics and robot control.

Lei Zeng was born in Jinggangshan, Jiangxi, China, in 1991. $\mathrm{He}$ received the B.Eng. degree from Nanchang University, Nanchang, China, in 2014. He is currently working toward the M.Sc. degree at the South China University of Technology, Guangzhou, China. His research interests include parallel mechanism control.

Xianmin Zhang was born in 1964. He received the Ph.D. degree from the Beijing University of Aeronautics and Astronautics, Beijing, China, in 1993. He has been a Professor with the South China University of Technology, Guangzhou, China, since 1998, where he was the Dean of the College of Mechanical and Automotive Engineering in 2013. His research interests include mechanism design and dynamics, as well as inspection technique. 